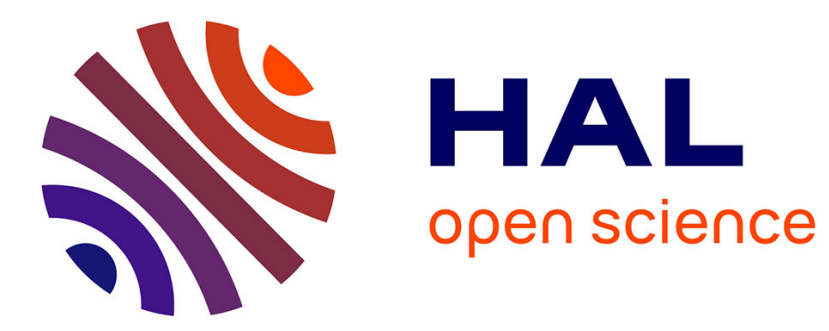

\title{
Asymptotic analysis of a linear isotropic elastic composite reinforced by a thin layer of periodically distributed isotropic parallel stiff fibres.
}

\author{
Michel Bellieud, Giuseppe Geymonat, Françoise Krasucki
}

\section{To cite this version:}

Michel Bellieud, Giuseppe Geymonat, Françoise Krasucki. Asymptotic analysis of a linear isotropic elastic composite reinforced by a thin layer of periodically distributed isotropic parallel stiff fibres.. Journal of Elasticity, 2016, 122, pp.43-74. 10.1007/s10659-015-9532-7 . hal-01121260

\author{
HAL Id: hal-01121260 \\ https://hal.science/hal-01121260
}

Submitted on 27 Feb 2015

HAL is a multi-disciplinary open access archive for the deposit and dissemination of scientific research documents, whether they are published or not. The documents may come from teaching and research institutions in France or abroad, or from public or private research centers.
L'archive ouverte pluridisciplinaire HAL, est destinée au dépôt et à la diffusion de documents scientifiques de niveau recherche, publiés ou non, émanant des établissements d'enseignement et de recherche français ou étrangers, des laboratoires publics ou privés. 


\title{
Asymptotic analysis of a linear isotropic elastic composite reinforced by a thin layer of periodically distributed isotropic parallel stiff fibres.
}

\author{
Michel Bellieud · Giuseppe Geymonat • Françoise \\ Krasucki
}

\begin{abstract}
We present some mathematical convergence results using a two-scale method for a linear elastic isotropic medium containing one layer of parallel periodically distributed heterogeneities located in the interior of the whole domain around a plane surface $\Sigma$. The aim of this paper is to study the situation when the rigidity of the linearly isotropic elastic fibres is $1 / \varepsilon^{m}$ the rigidity of the surrounding linearly isotropic elastic material. We use a two-scale convergence method adapted to the geometry of the problem (layer of fibres). In the models obtained $\Sigma$ behaves for $m=1$ as a "material surface" without membrane energy in the direction of the plane orthogonal to the direction of the fibres. For $m=3$ the "material surface" has no bending energy in the direction orthogonal to the fibres.
\end{abstract}

AMS subject classifications: 35B27, 35B40, 49J45, 74A40, 74Q05

Keywords: linear elasticity, fibres reinforced structures, two-scale convergence, homogenization

\section{Contents}

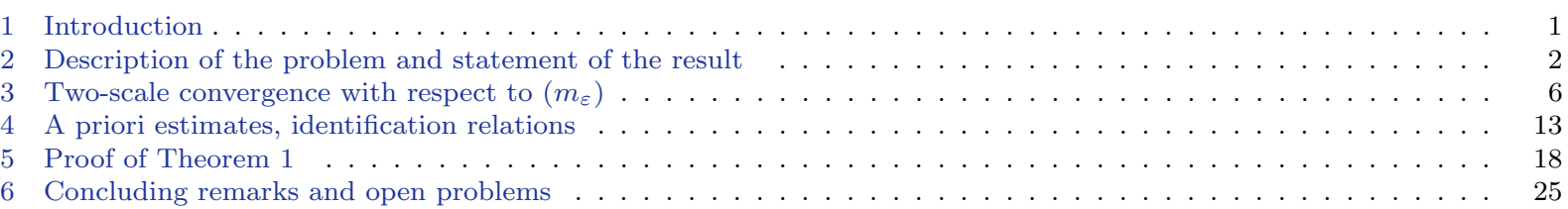

\section{Introduction}

Due to its importance for many applied problems, the study of boundary value problems where the domain and/or the coefficients have a large number of heterogeneities has attracted the interest of many researchers of the mathematical, of the physical and of the engineering communities. Hence the corresponding literature is huge. When the heterogeneities (inclusions, holes, layers, fibres) are periodically

Michel Bellieud

LMGC (Laboratoire de Mécanique et de Génie Civil de Montpellier) UMR-CNRS 5508, Université Montpellier II, Case courier 048, Place Eugène Bataillon, 34095 Montpellier Cedex 5, France

E-mail: michel.bellieud@univ-montp2.fr

Giuseppe Geymonat

Laboratoire de Mécanique des Solides, UMR-CNRS 7649,

École Polytechnique

91128 PALAISEAU Cedex, France

E-mail: giuseppe.geymonat@lms.polytechnique.fr

Françoise Krasucki

I3M, UMR-CNRS 5149 , Université Montpellier II, Case courier 051,

Place Eugène Bataillon, 34095 Montpellier Cedex 5, France

E-mail: krasucki@math.univ-montp2.fr 
distributed in all the volume (the ratio between a characteristic size of the period and a characteristic size of the whole structure being traditionally denoted $\varepsilon$ ) and (still with respect to the whole structure) the characteristic size of the heterogeneities is a power of $\varepsilon$, in order to obtain numerically efficient (i.e. precise and not too much computationally expensive) simplified models, it is now classical to use an homogenization method. An advantage of the homogenization methods is that they have been developed and fully mathematically justified for many different geometrical and/or mechanical situations (in particular when also the ratio between the mechanical characteristics of the heterogeneities and of the surrounding material can depend on $\varepsilon$ ), see, e.g., [10], [32], [21], [30], [31], [7], [16], and also [14,27,33] in the context of nonlinear elasticity.

Another situation of interest for the engineering community arises when there exists only one layer of heterogeneity located in the interior of the whole domain. This is for instance the situation when two bodies of different (or equal) nature are pasted together using an homogeneous thin layer "centred" around a surface $\Sigma$ and whose transversal depth has a ratio to the whole structure generally denoted $\varepsilon$. In this case many numerically efficient simplified models have been constructed and mathematically fully justified for various mechanical and /or geometrical circumstances; see, e.g., [17], [20], [11], [13], and also $[14,27,33]$ in the context of nonlinear elasticity.

Still another situation arises when the heterogeneities are periodically distributed in a thin layer "centred" around a surface $\Sigma$. In some cases of inclusions one can use matched asymptotic expansion methods (see, e.g., [29], [1], [26], ...). The aim of this paper is to study the situation when the heterogeneities in the layer "centred" around a surface $\Sigma$ are linearly elastic isotropic parallel fibres periodically distributed and whose rigidity is higher with respect of the rigidity of the surrounding linearly elastic material.

In Section 2 we describe the problem and we state the main results; in Section 3 we introduce the notion of two-scale convergence with respect to a family of measures $m_{\varepsilon}$ essentially connected with the total volume of the fibres. This is a variant of the two-scale convergence introduced by Nguetseng [28] and developed by G. Allaire in [2]. In Section 4 we prove the a priori estimates and we identify the limits obtained with the two-scale method; these results are fundamental for the proof of the weak convergence (Section 5.1) and the strong convergence (Section 5.2).

Notations. In the sequel, $\left\{\boldsymbol{e}_{1}, \boldsymbol{e}_{2}, \boldsymbol{e}_{3}\right\}$ stands for the canonical basis of $\mathbb{R}^{3}$. Points in $\mathbb{R}^{3}$ or in $\mathbb{Z}^{3}$ and real-valued functions are represented by symbols beginning by a light-face minuscule (example $x, i, \operatorname{det} \boldsymbol{A} \ldots$ ), vectors and vector-valued functions by symbols beginning by a boldface minuscule (examples: $\left.\boldsymbol{x}, \boldsymbol{x}_{S}, \boldsymbol{i}, \boldsymbol{u}, \boldsymbol{f}, \boldsymbol{g}, \boldsymbol{\operatorname { d i v }} \boldsymbol{\Psi}, \ldots\right)$. Matrices and matrix-valued functions are represented by symbols beginning by a capital boldface with the following exceptions: $\boldsymbol{\nabla} \boldsymbol{u}$ (displacement gradient), $\boldsymbol{e}(\boldsymbol{u})$ (linearised strain tensor). We denote by $u_{i}$ or $(\boldsymbol{u})_{i}$ the components of a vector $\boldsymbol{u}$ and by $A_{i j}$ or $(\boldsymbol{A})_{i j}$ those of a matrix $\boldsymbol{A}$ (that is $\left.\boldsymbol{u}=\sum_{i=1}^{3} u_{i} \boldsymbol{e}_{i}=\sum_{i=1}^{3}(\boldsymbol{u})_{i} \boldsymbol{e}_{i} ; \boldsymbol{A}=\sum_{i, j=1}^{3} A_{i j} \boldsymbol{e}_{i} \otimes \boldsymbol{e}_{j}=\sum_{i, j=1}^{3}(\boldsymbol{A})_{i j} \boldsymbol{e}_{i} \otimes \boldsymbol{e}_{j}\right) .{ }^{t} \boldsymbol{A}$ denotes the transposed matrix of $\boldsymbol{A}$. We do not employ the usual repeated index convention for summation. We denote by $\boldsymbol{A}: \boldsymbol{B}=\sum_{i, j=1}^{3} A_{i j} B_{i j}$ the inner product of two matrices, by $\mathbb{S}^{N}$ the set of all real symmetric matrices of order $N$, and by $\boldsymbol{I}_{N}$ the $N \times N$ identity matrix. The symbols $\mathcal{L}^{N}$ and $\mathcal{H}^{k}$ represent, respectively, the Lebesgue outer measure on $\mathbb{R}^{N}$ and the $k$-dimensional Hausdorff outer measure on $\mathbb{R}^{N}$. For any $x=\left(x_{1}, x_{2}, x_{3}\right) \in \mathbb{R}^{3}$, we set $x^{\prime}=\left(x_{1}, x_{2}\right)$. Given an open subset $A$ of $\mathbb{R}^{2}$ we note $f_{A} f d y$ the mean value of $\mathbf{f}$ on $A$. Given an open subset $\Omega$ of $\mathbb{R}^{3}$ and a 2-dimensional Lipschitz sub-manifold $\Sigma$ of $\bar{\Omega}$, the trace on $\Sigma$ of an element $\varphi$ of $H^{1}\left(\Omega ; \mathbb{R}^{k}\right)$ is denoted by $\gamma_{\Sigma}(\varphi)$ (resp. $\gamma_{\Sigma}(\varphi)$ if $k=1$ ), or occasionally by the same symbol $\varphi$ when ambiguity is not possible. If $A$ is a subset of $\Omega$, the symbol $\mathbb{1}_{A}$ represents the characteristic function of $A$. The letter $C$ denotes different constants whose precise values may vary.

\section{Description of the problem and statement of the result}

Let $\Omega:=(-L, L)^{3}$, let $S$ be a bounded Lipschitz domain of $\mathbb{R}^{2}$ satisfying

$$
D \subset S \subset \bar{S} \subset Y, \quad Y:=\left(-\frac{1}{2}, \frac{1}{2}\right)^{2}, \quad f_{S} \boldsymbol{y} d y=0,
$$

for some open disk $D$ of $\mathbb{R}^{2}$ centred at the origin and let $T:=S \times(-L, L)$. For $\varepsilon>0$ we set (see fig. 1):

$$
\begin{aligned}
& Y_{\varepsilon}^{i}:=\varepsilon((0, i)+Y), \quad I_{\varepsilon}:=\left\{i \in \mathbb{Z}, Y_{\varepsilon}^{i} \times(-L, L) \subset \Omega\right\} \\
& S_{\varepsilon}^{i}:=\varepsilon((0, i)+S), \quad T_{\varepsilon}^{i}=S_{\varepsilon}^{i} \times(-L, L), \quad T_{\varepsilon}=\bigcup_{i \in I_{\varepsilon}} T_{\varepsilon}^{i},
\end{aligned}
$$

$i^{\min }:=\min I_{\varepsilon}, \quad i^{\max }:=\max I_{\varepsilon}$,

$\Sigma:=\Omega \cap\{0\} \times \mathbb{R}^{2}, \quad \Sigma^{\varepsilon}:=\Sigma \cap T_{\varepsilon}=\left\{x \in \Sigma, \quad \varepsilon i^{\text {min }}-\frac{\varepsilon}{2}<x_{2}<\varepsilon i^{\text {max }}+\frac{\varepsilon}{2}\right\}$,

$\Omega^{-}:=\Omega \cap\left((-\infty, 0) \times \mathbb{R}^{2}\right), \quad \Omega^{+}:=\Omega \cap\left((0,+\infty) \times \mathbb{R}^{2}\right)$. 
Let us remark that

$$
\mathcal{L}^{3}\left(T_{\varepsilon}\right) \simeq \varepsilon|S||\Sigma| \quad \text { as } \quad \varepsilon \rightarrow 0
$$

where, for simplicity, $|S|$ (resp. $|\Sigma|$ ) denotes the area, that is the two-dimensional Hausdorff measure of $S$ (resp. $\Sigma$ ). Hence from a geometrical point of view there are two natural length scales: the first is a global one (i.e. the $3 D$-diameter of $\Omega$ ) the other one is a local one connected with the heterogeneities (the diameter of the cross section of every fiber). The ratio between these two scales will be denoted by $\varepsilon$. More precisely, the parameter $\varepsilon$ is a non-dimensional parameter characterizing the geometrical distribution of the heterogeneities in the structure since, at the same time, it characterizes the ratio between the diameter of the cross section of the fibres and the diameter of $\Omega$ and the ratio between the diameter of the cross section of the fibres and the length in the transversal direction $\boldsymbol{e}_{2}$ of the planar set $\Sigma$ supporting the heterogeneities.

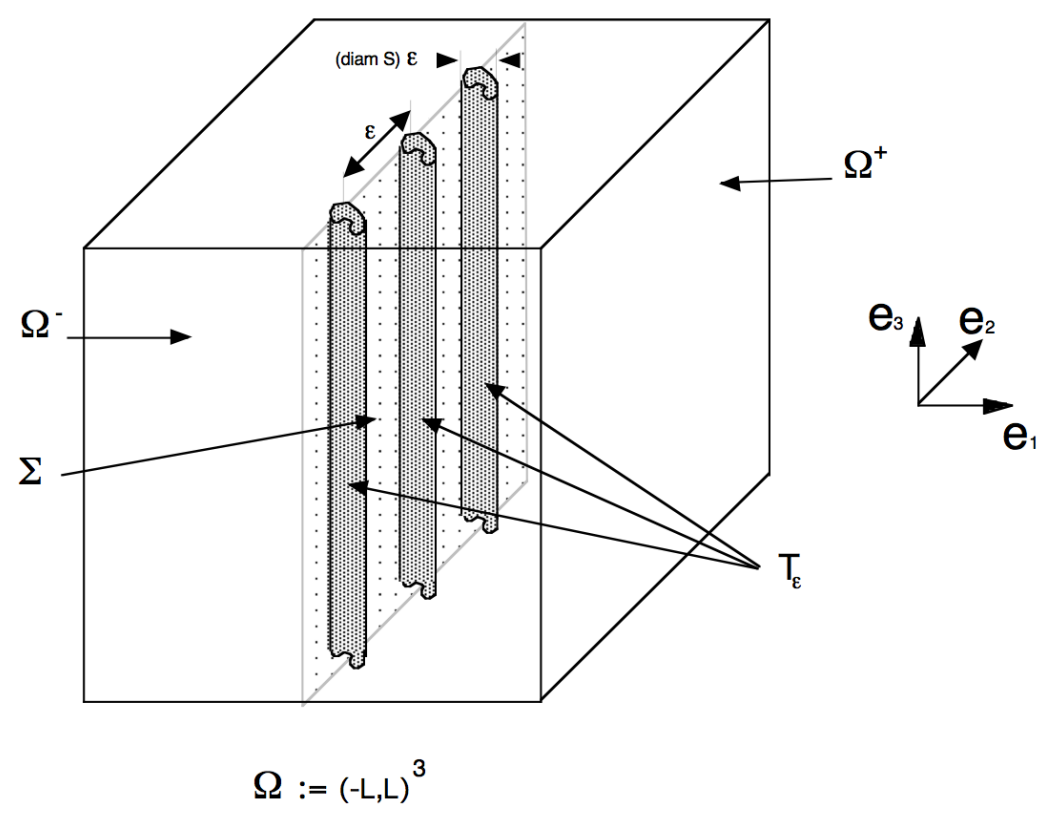

Fig. 1 The layer of fibres

We consider the case of a linear isotropic elastic structure occupying the set $\Omega$ reinforced by the linear isotropic elastic parallel fibres $T_{\varepsilon}^{i}$ of very high stiffness surrounded by a matrix of constant stiffness. More precisely we assume that the Young's modulus $E_{\varepsilon}$ depends on $\varepsilon$ as follows :

$$
\begin{cases}E_{\varepsilon}(x)=\frac{1}{\varepsilon^{m}} E_{T} & \text { if } x \in T_{\varepsilon}, \\ E_{\varepsilon}(x)=E & \text { if } x \in \Omega \backslash T_{\varepsilon},\end{cases}
$$

with $m>0$. Instead the Poisson's coefficient denoted by $\nu_{\varepsilon}$ is independent from $\varepsilon$ :

$$
\begin{cases}\nu_{\varepsilon}(x)=\nu_{1} & \text { if } x \in T_{\varepsilon}, \\ \nu_{\varepsilon}(x)=\nu & \text { if } x \in \Omega \backslash T_{\varepsilon} .\end{cases}
$$

We also assume that they satisfy the usual conditions $E, E_{T}>0$ and $0<\nu, \nu_{1}<\frac{1}{2}$. Let us stress that, with this choice, the parameter $\varepsilon$ characterizes at the same time the geometrical distribution of the heterogeneities in the structure and the ratio between the rigidity of the heterogeneities and the rigidity 
of the structure. We are concerned with the behaviour for $\varepsilon \longrightarrow 0$ of the following elasticity problem where we have taken for simplicity homogeneous Dirichlet boundary data:

$$
\left\{\begin{array}{l}
-\operatorname{div} \boldsymbol{\sigma}_{\varepsilon}=\boldsymbol{f} \quad \text { in } \quad \Omega \\
\boldsymbol{u}_{\varepsilon} \in H_{0}^{1}\left(\Omega, \mathbb{R}^{3}\right), \quad \boldsymbol{f} \in L^{2}\left(\Omega, \mathbb{R}^{3}\right), \\
\boldsymbol{\sigma}_{\varepsilon}=\frac{E_{\varepsilon}}{1+\nu_{\varepsilon}}\left(\frac{\nu_{\varepsilon}}{1-2 \nu_{\varepsilon}} \operatorname{tr}\left(\boldsymbol{e}\left(\boldsymbol{u}_{\varepsilon}\right)\right) \boldsymbol{I}+\boldsymbol{e}\left(\boldsymbol{u}_{\varepsilon}\right)\right), \quad \boldsymbol{e}\left(\boldsymbol{u}_{\varepsilon}\right)=\frac{1}{2}\left(\boldsymbol{\nabla} \boldsymbol{u}_{\varepsilon}+{ }^{t} \boldsymbol{\nabla} \boldsymbol{u}_{\varepsilon}\right) .
\end{array}\right.
$$

The limit problem we derive in Theorem 1 depends on the value of $m$.

Case $0<m<1$. The limit problem reads:

$$
\begin{cases}-\operatorname{div} \sigma_{0}=f & \text { in } \Omega, \\ \boldsymbol{\sigma}_{0}=\frac{E}{1+\nu}\left(\frac{\nu}{1-2 \nu} \operatorname{tr}(\boldsymbol{e}(\boldsymbol{u})) \boldsymbol{I}+\boldsymbol{e}(\boldsymbol{u})\right) & \\ \boldsymbol{u} \in \mathcal{D}_{m} & \end{cases}
$$

where

$$
\mathcal{D}_{m}=H_{0}^{1}\left(\Omega ; \mathbb{R}^{3}\right)
$$

Case $m=1$. The limit problem reads:

$$
\left\{\begin{array}{lr}
-\operatorname{div} \boldsymbol{\sigma}_{0}=\boldsymbol{f} & \text { in } \Omega^{+} \cup \Omega^{-}, \\
\boldsymbol{\sigma}_{0}=\frac{E}{1+\nu}\left(\frac{\nu}{1-2 \nu} \operatorname{tr}(\boldsymbol{e}(\boldsymbol{u})) \boldsymbol{I}+\boldsymbol{e}(\boldsymbol{u})\right), & \\
\left(\boldsymbol{\sigma}_{0}^{+}-\boldsymbol{\sigma}_{0}^{-}\right) \boldsymbol{e}_{1}+|S| E_{T} \frac{\partial^{2} \gamma_{\Sigma}\left(u_{3}\right)}{\partial x_{3}^{2}} \boldsymbol{e}_{3}=0 & \text { on } \Sigma, \\
\boldsymbol{u} \in \mathcal{D}_{1}, &
\end{array}\right.
$$

where $\boldsymbol{\sigma}_{0}^{-}$(resp. $\boldsymbol{\sigma}_{0}^{+}$) denotes the restriction of $\boldsymbol{\sigma}_{0}$ to $\Omega^{-}\left(\right.$resp. $\Omega^{+}$) and

$$
\mathcal{D}_{1}=\left\{\boldsymbol{u} \in H_{0}^{1}\left(\Omega ; \mathbb{R}^{3}\right) ; \frac{\partial \gamma_{\Sigma}\left(u_{3}\right)}{\partial x_{3}} \in L^{2}(\Sigma), \quad \gamma_{\Sigma}\left(u_{3}\right)=0 \text { on } \partial \Sigma \cap\left\{x \in \mathbb{R}^{3}, x_{3} \in\{-L ; L\}\right\}\right\} .
$$

Note that the effective superficial density of forces exerted by the fibres on the matrix along $\Sigma$, given by

$$
\left(\boldsymbol{\sigma}_{0}^{+}-\boldsymbol{\sigma}_{0}^{-}\right) \boldsymbol{e}_{1}=-|S| E_{T} \frac{\partial^{2} \gamma_{\Sigma}\left(u_{3}\right)}{\partial x_{3}^{2}} \boldsymbol{e}_{3},
$$

is parallel to the fibres.

Case $1<m<3$. The limit problem reads:

$$
\begin{cases}-\operatorname{div} \sigma_{0}=f & \text { in } \Omega^{+} \cup \Omega^{-}, \\ \sigma_{0}=\frac{E}{1+\nu}\left(\frac{\nu}{1-2 \nu} \operatorname{tr}(\boldsymbol{e}(\boldsymbol{u})) \boldsymbol{I}+\boldsymbol{e}(\boldsymbol{u})\right), & \\ \boldsymbol{u} \in \mathcal{D}_{m} & \end{cases}
$$

where

$$
\mathcal{D}_{m}=\left\{\boldsymbol{u} \in H_{0}^{1}\left(\Omega ; \mathbb{R}^{3}\right) ; \quad \gamma_{\Sigma}\left(u_{3}\right)=0\right\} .
$$


Case $m=3$. The limit problem reads:

$$
\begin{cases}-\operatorname{div} \boldsymbol{\sigma}_{0}=\boldsymbol{f} & \text { in } \Omega^{+} \cup \Omega^{-}, \\ \boldsymbol{\sigma}_{0}=\frac{E}{1+\nu}\left(\frac{\nu}{1-2 \nu} \operatorname{tr}(\boldsymbol{e}(\boldsymbol{u})) \boldsymbol{I}+\boldsymbol{e}(\boldsymbol{u})\right) & \\ \left(\boldsymbol{\sigma}_{0}^{-}-\boldsymbol{\sigma}_{0}^{+}\right) \boldsymbol{e}_{1}+|S| E_{T} \sum_{\alpha, \beta=1}^{2} J_{\alpha \beta} \frac{\partial^{4} \gamma_{\Sigma}\left(u_{\beta}\right)}{\partial x_{3}^{4}} \boldsymbol{e}_{\alpha}=0 & \text { on } \Sigma \\ \boldsymbol{u} \in \mathcal{D}_{3}, & \end{cases}
$$

where

$$
J_{\alpha \beta}:=f_{S} y_{\alpha} y_{\beta} d y
$$

and

$$
\mathcal{D}_{3}=\left\{\boldsymbol{u} \in H_{0}^{1}\left(\Omega ; \mathbb{R}^{3}\right) ; \begin{array}{c}
\gamma_{\Sigma}\left(u_{3}\right)=0, \quad \frac{\partial^{2} \gamma_{\Sigma}\left(u_{1}\right)}{\partial x_{3}^{2}}, \frac{\partial^{2} \gamma_{\Sigma}\left(u_{2}\right)}{\partial x_{3}^{2}} \in L^{2}(\Sigma) \\
\gamma_{\Sigma}\left(u_{\alpha}\right)=\frac{\partial \gamma_{\Sigma}\left(u_{\alpha}\right)}{\partial x_{3}}=0 \text { on } \Sigma \cap\left\{x \in \mathbb{R}^{3}, x_{3} \in\{-L ; L\}\right\} \forall \alpha \in\{1,2\}
\end{array}\right\}
$$

Unlike the case $m=1$, the effective superficial density of forces exerted by the fibres on the matrix along $\Sigma$, given by

$$
\boldsymbol{h}=\left(\boldsymbol{\sigma}_{0}^{+}-\boldsymbol{\sigma}_{0}^{-}\right) \boldsymbol{e}_{1}=|S| E_{1} \sum_{\alpha, \beta=1}^{2} J_{\alpha \beta} \frac{\partial^{4} \gamma_{\Sigma}\left(u_{\beta}\right)}{\partial x_{3}^{4}} \boldsymbol{e}_{\alpha},
$$

is orthogonal to the direction of the fibres.

Case $m>3$. The limit problem reads:

$$
\begin{cases}-\operatorname{div} \sigma_{0}=f & \text { in } \Omega^{+} \cup \Omega^{-} \\ \boldsymbol{\sigma}_{0}=\frac{E}{1+\nu}\left(\frac{\nu}{1-2 \nu} \operatorname{tr}(\boldsymbol{e}(\boldsymbol{u})) \boldsymbol{I}+\boldsymbol{e}(\boldsymbol{u})\right) & \\ \boldsymbol{u} \in \mathcal{D}_{m} & \end{cases}
$$

where

$$
\mathcal{D}_{m}=\left\{\boldsymbol{u} \in H_{0}^{1}\left(\Omega ; \mathbb{R}^{3}\right) ; \quad \gamma_{\Sigma}(\boldsymbol{u})=0\right\} .
$$

Theorem 1 Assume (5) and (6), then for $\varepsilon \rightarrow 0$ the solution $\boldsymbol{u}_{\varepsilon}$ of (7) strongly converges in $H_{0}^{1}\left(\Omega ; \mathbb{R}^{3}\right)$ ) to the unique solution $\boldsymbol{u}$ of (8) if $0<m<1$, of (10) if $m=1$, of (12) if $1<m<3$, of (14) if $m=3$, and of (17) if $m>3$.

Remark 1 For every $m>0$ the limit problem does not depend on the value $\nu_{1}$ of the Poisson's coefficient of the material of the fibres.

The problem (7) has a unique solution $\boldsymbol{u}_{\varepsilon}$ that realizes the minimum in $H_{0}^{1}\left(\Omega, \mathbb{R}^{3}\right)$ of the functional

$$
\mathcal{F}_{\varepsilon, m}(\boldsymbol{v}):=\frac{1}{2} a_{\varepsilon, m}(\boldsymbol{v}, \boldsymbol{v})-\int_{\Omega} \boldsymbol{f} . \boldsymbol{v} d x,
$$

with

$$
\frac{1}{2} a_{\varepsilon, m}(\boldsymbol{v}, \boldsymbol{v}):=\frac{1}{2} a_{\varepsilon}(\boldsymbol{v}, \boldsymbol{v})+\frac{1}{2 \varepsilon^{m}} a_{\varepsilon, T}(\boldsymbol{v}, \boldsymbol{v})
$$


where $a_{\varepsilon}(.,$.$) and a_{\varepsilon, T}(.,$.$) are the symetric bilinear forms on H_{0}^{1}\left(\Omega, \mathbb{R}^{3}\right) \times H_{0}^{1}\left(\Omega, \mathbb{R}^{3}\right)$ given by :

$$
\begin{aligned}
& a_{\varepsilon}(\boldsymbol{v}, \boldsymbol{\phi})=\int_{\Omega \backslash T_{\varepsilon}}\left\{\frac{E}{1+\nu}\left(\frac{\nu}{1-2 \nu} \operatorname{tr}(\boldsymbol{e}(\boldsymbol{v})) \boldsymbol{I}+\boldsymbol{e}(\boldsymbol{v})\right): \boldsymbol{e}(\boldsymbol{\phi})\right\} d x, \\
& a_{\varepsilon, T}(\boldsymbol{v}, \boldsymbol{\phi})=\int_{T_{\varepsilon}}\left\{\frac{E_{T}}{1+\nu_{1}}\left(\frac{\nu_{1}}{1-2 \nu_{1}} \operatorname{tr}(\boldsymbol{e}(\boldsymbol{v})) \boldsymbol{I}+\boldsymbol{e}(\boldsymbol{v})\right): \boldsymbol{e}(\boldsymbol{\phi})\right\} d x .
\end{aligned}
$$

Let us point out that the limit problems have a unique solution $\boldsymbol{u} \in \mathcal{D}_{m}$ that realizes the minimum in $\mathcal{D}_{m}$ of the functional $\mathcal{F}_{m}$ defined on $\mathcal{D}_{m}$ by

$$
\mathcal{F}_{m}(\boldsymbol{v}):=\frac{1}{2} a_{m}(\boldsymbol{v}, \boldsymbol{v})-\int_{\Omega} \boldsymbol{f} . \boldsymbol{v}
$$

where $a_{m}(.,$.$) is the bilinear form on \mathcal{D}_{m} \times \mathcal{D}_{m}$ given by:

$$
\begin{aligned}
& a_{0}(\boldsymbol{v}, \boldsymbol{\phi}):=\int_{\Omega} \frac{E}{1+\nu}\left(\frac{\nu}{1-2 \nu} \operatorname{tr}(\boldsymbol{e}(\boldsymbol{v})) \boldsymbol{I}+\boldsymbol{e}(\boldsymbol{v})\right): \boldsymbol{e}(\boldsymbol{\phi}) d x, \\
& a_{1}(\boldsymbol{v}, \boldsymbol{\phi}):=a_{0}(\boldsymbol{v}, \boldsymbol{\phi})+|S| E_{T} \int_{\Sigma} \frac{\partial \gamma_{\Sigma}\left(v_{3}\right)}{\partial x_{3}} \frac{\partial \gamma_{\Sigma}\left(\phi_{3}\right)}{\partial x_{3}} d \mathcal{H}^{2}, \\
& a_{3}(\boldsymbol{v}, \boldsymbol{\phi}):=a_{0}(\boldsymbol{v}, \boldsymbol{\phi})+|S| E_{T} \sum_{\alpha, \beta=1}^{2} \int_{\Sigma} J_{\alpha \beta} \frac{\partial^{2} \gamma_{\Sigma}\left(v_{\alpha}\right)}{\partial x_{3}^{2}} \frac{\partial^{2} \gamma_{\Sigma}\left(\phi_{\beta}\right)}{\partial x_{3}^{2}} d \mathcal{H}^{2}(x), \\
& a_{m}(\boldsymbol{v}, \boldsymbol{\phi}):=a_{0}(\boldsymbol{v}, \boldsymbol{\phi}) \quad \text { if } m \in(0,+\infty) \backslash\{1 ; 3\} .
\end{aligned}
$$

The proof of Theorem 1 is structured as follows:

i) In Sect.3, we introduce a two-scale convergence method adapted to the particular geometry of our problem (layer of fibres) and we prove some useful properties of this method.

ii) In Sect.4, we study the behaviour when $\varepsilon \rightarrow 0$ of the solution $\left(\boldsymbol{u}_{\varepsilon}\right)$ of (7). The most delicate task resides in the study of the behaviour of $\left(\boldsymbol{u}_{\varepsilon}\right)$ in the fibres and here we use the properties of the two-scale method introduced in Sect.3

iii) In Sect.5.1, we multiply (7) by an appropriate sequence of oscillating test fields $\left(\phi_{\varepsilon}\right)$ and, by passing to the limit as $\varepsilon \rightarrow 0$ in accordance with the convergences established in ii), we obtain a variational formulation of the limit problem satisfied by $\boldsymbol{u}$.

iv) We prove in Sect.5.2 the strong convergence using the linearity of the problem.

\section{Two-scale convergence with respect to $\left(m_{\varepsilon}\right)$}

As a mean to particularize the oscillatory behaviour of the displacement in the fibres, we consider a variant of the two-scale convergence introduced by G. Nguetseng in [28]. This seminal idea has been further developed by G. Allaire in [2] (see also a more general presentation in [22]) and extended in various ways (e.g. for the two-scale convergence with respect to a sequence of measures see [8], [23]), [36],...). Here the two-scale convergence is adapted to the geometry of the problem i.e. that the heterogeneities are a layer of fibres.

Let us at first introduce the following shortened notation for $\mathbb{R}$-valued functions of $L^{1}(\Omega)$ (the analogous notation will also be used for $\mathbb{R}^{k}$-valued functions):

$$
\int f(x) d m_{\varepsilon}:=\frac{1}{\varepsilon|S|} \int_{T_{\varepsilon}} f\left(x_{1}, x_{2}, x_{3}\right) d x_{1} d x_{2} d x_{3}=\frac{1}{\varepsilon|S|} \sum_{i \in I_{\varepsilon}} \int_{-L}^{L} d x_{3} \int_{S_{\varepsilon}^{i}} f\left(x_{1}, x_{2}, x_{3}\right) d x_{1} d x_{2},
$$

where $|S|$ denotes the two-dimensional Lebesgue measure of $S$. Notice that by (4) there holds

$$
\int d m_{\varepsilon} \simeq|\Sigma|
$$


In the study of the behaviour when $\varepsilon \rightarrow 0$ of the solution $\left(\boldsymbol{u}_{\varepsilon}\right)$ of $(7)$ a delicate task resides in the study of the behaviour of the fibres. For this we use the operator $\mathfrak{v}_{\varepsilon}: L^{2}(\Omega) \rightarrow L^{2}(\Omega)$ defined by

$$
\mathfrak{v}_{\varepsilon}(\varphi)(x):=\sum_{i \in I_{\varepsilon}}\left(f_{S_{\varepsilon}^{i}} \varphi\left(s, x_{3}\right) d \mathcal{H}^{2}(s)\right) \mathbb{1}_{Y_{\varepsilon}^{i}}\left(x_{1}, x_{2}\right)
$$

(in the case of $\mathbb{R}^{k}$-valued functions the analogous definition will be used). We also define the $\mathbb{R}^{2}$-valued function (see (2))

$$
y_{\varepsilon}\left(x^{\prime}\right)=y_{\varepsilon}\left(x_{1}, x_{2}\right):=\sum_{i \in I_{\varepsilon}}\left(x_{1} \boldsymbol{e}_{1}+\left(x_{2}-\varepsilon i\right) \boldsymbol{e}_{2}\right) \mathbb{1}_{Y_{\varepsilon}^{i}}\left(x_{1}, x_{2}\right)
$$

A sequence $\left(\boldsymbol{f}_{\varepsilon}\right)$ in $L^{2}\left(\Omega ; \mathbb{R}^{k}\right)$ will be said to two-scale converge with respect to $\left(m_{\varepsilon}\right)$ to some $\boldsymbol{f}_{0} \in$ $L^{2}\left(\Sigma \times S ; \mathbb{R}^{k}\right)$ (notation: $\left.\boldsymbol{f}_{\varepsilon} \stackrel{m_{\varepsilon}}{\boldsymbol{f}_{0}}\right)$ if for all $\boldsymbol{\psi} \in C\left(\overline{\Omega \times S} ; \mathbb{R}^{k}\right)$

$$
\lim _{\varepsilon \rightarrow 0} \int \boldsymbol{f}_{\varepsilon}(x) \cdot \boldsymbol{\psi}\left(x, \frac{y_{\varepsilon}\left(x^{\prime}\right)}{\varepsilon}\right) d m_{\varepsilon}(x)=\frac{1}{|S|} \int_{\Sigma \times S} \boldsymbol{f}_{0}\left(x_{2}, x_{3} ; y_{1}, y_{2}\right) \cdot \boldsymbol{\psi}\left(0, x_{2}, x_{3} ; y_{1}, y_{2}\right) d \mathcal{H}^{2}(x) d y
$$

where $y_{\varepsilon}\left(x^{\prime}\right)$ is given by $(26)$ and $|S|$ denotes the two-dimensional Lebesgue measure of $S$. Similar notions have been considered in [8], [23], [36]. As shown in the next proposition, the two-scale convergence with respect to $\left(m_{\varepsilon}\right)$ enjoys a compactness property for sequences satisfying an uniform bound of the type $\sup _{\varepsilon>0} \int\left|\boldsymbol{f}_{\varepsilon}\right|^{2} d m_{\varepsilon} \leq C$.

\section{Proposition 1 (i) There holds}

$$
\lim _{\varepsilon \rightarrow 0} \int \boldsymbol{\psi}\left(x, \frac{y_{\varepsilon}\left(x^{\prime}\right)}{\varepsilon}\right) d m_{\varepsilon}(x)=\frac{1}{|S|} \int_{\Sigma \times S} \boldsymbol{\psi}(x, y) d \mathcal{H}^{2}(x) d y \quad \forall \boldsymbol{\psi} \in C\left(\overline{\Omega \times S} ; \mathbb{R}^{k}\right) .
$$

(ii) Let $\left(\boldsymbol{f}_{\varepsilon}\right)$ be a sequence in $L^{2}\left(\Omega ; \mathbb{R}^{k}\right)$ such that $\sup _{\varepsilon>0} \int\left|\boldsymbol{f}_{\varepsilon}\right|^{2} d m_{\varepsilon}<+\infty$. Then, the sequence $\left(\boldsymbol{f}_{\varepsilon}\right)$ two-scale converges with respect to $\left(m_{\varepsilon}\right)$, up to a subsequence, to some $\boldsymbol{f}_{0} \in L^{2}\left(\Sigma \times S ; \mathbb{R}^{k}\right)$.

(iii) Let $\left(\boldsymbol{f}_{\varepsilon}\right)$ be a sequence in $L^{2}\left(\Omega ; \mathbb{R}^{k}\right)$ such that $\sup _{\varepsilon>0} \int\left|\boldsymbol{f}_{\varepsilon}\right|^{2} d m_{\varepsilon}<+\infty$ and that $\left(\boldsymbol{f}_{\varepsilon}\right)$ two-scale converges with respect to $\left(m_{\varepsilon}\right)$ to $f_{0} \in L^{2}\left(\Sigma \times S ; \mathbb{R}^{k}\right)$. Then:

a) the sequence $\left(\boldsymbol{v}_{\varepsilon}\left(\boldsymbol{f}_{\varepsilon}\right)\right)$ two-scale converges with respect to $\left(m_{\varepsilon}\right)$ to $\frac{1}{|S|} \int_{S} \boldsymbol{f}_{0}(x, y) d y$;

b) the sequence of traces $\left(\boldsymbol{\gamma}_{\Sigma}\left(\boldsymbol{v}_{\varepsilon}\left(\boldsymbol{f}_{\varepsilon}\right)\right)\right)$ is well defined and weakly converges in $L^{2}\left(\Sigma ; \mathbb{R}^{k}\right)$ to $\frac{1}{|S|} \int_{S} \boldsymbol{f}_{0}(x, y) d y$;

c) if $\boldsymbol{f}_{\varepsilon}$ takes constant values for a.e. $x_{3} \in(-L, L)$ on each set $Y_{\varepsilon}^{i} \times\left\{x_{3}\right\}$, and vanishes elsewhere, then the sequence of traces $\left(\boldsymbol{\gamma}_{\Sigma}\left(\boldsymbol{f}_{\varepsilon}\right)\right)$ is well defined, $\boldsymbol{f}_{\varepsilon}=\boldsymbol{v}_{\varepsilon}\left(\boldsymbol{f}_{\varepsilon}\right), \boldsymbol{f}_{0}(x, y)=f_{S} \boldsymbol{f}_{0}\left(x, y^{\prime}\right) d y^{\prime}$ a.e. on $\Sigma \times S$, and $\left(\boldsymbol{\gamma}_{\Sigma}\left(\boldsymbol{f}_{\varepsilon}\right)\right)$ weakly converges in $L^{2}\left(\Sigma ; \mathbb{R}^{k}\right)$, up to a subsequence, to $\frac{1}{|S|} \int_{S} \boldsymbol{f}_{0}(x, y) d y$.

Proof. ${ }^{1}$ In analogy to [3,27,33,35] (and in the spirit of the periodic unfolding method [15]), denoting by $[s]$ the integer part of a real $s$, we define the transformation $S_{\varepsilon}: \Sigma^{\varepsilon} \times S \rightarrow T_{\varepsilon}$ as follows

$$
S_{\varepsilon}(x, y):=\left(\varepsilon y_{1}, \varepsilon\left[x_{2} / \varepsilon+1 / 2\right]+\varepsilon y_{2}, x_{3}\right) .
$$

For arbitrary $f \in L^{1}\left(T_{\varepsilon}\right)$ the function $\left(f \circ S_{\varepsilon}\right)(x, y) \in L^{1}\left(\Sigma^{\varepsilon} \times S\right)$. Its extension by zero to $\Sigma \times S$ will be indicated $f_{\varepsilon} \circ S_{\varepsilon} \mathbb{1}_{\Sigma^{\varepsilon} \times S}$ or simply $\left(f \circ S_{\varepsilon}\right)$ when there are no ambiguities. By the following isometry property

$$
\int f d m_{\varepsilon}:=\frac{1}{\varepsilon|S|} \int_{T_{\varepsilon}} f d x=\frac{1}{|S|} \int_{\Sigma^{\varepsilon} \times S}\left(f \circ S_{\varepsilon}\right)(x, y) d \mathcal{H}^{2}(x) d y,
$$

any sequence $f_{\varepsilon} \in L^{2}\left(T_{\varepsilon}\right)$ with $\sup _{\varepsilon>0} \int\left|f_{\varepsilon}\right|^{2} d m_{\varepsilon}<+\infty$ yields a subsequence with

$$
f_{\varepsilon} \circ S_{\varepsilon} \mathbb{1}_{\Sigma^{\varepsilon} \times S} \rightarrow f_{0} \quad \text { weakly in } L^{2}(\Sigma \times S) .
$$

\footnotetext{
1 We thank an anonymous referee for his/her suggestions who permitted to notably simplify the original proof.
} 
If $\psi \in C(\overline{\Omega \times S})$ and $\psi_{\varepsilon}(x):=\psi\left(x, \frac{y_{\varepsilon}\left(x^{\prime}\right)}{\varepsilon}\right)$ then

$$
\psi_{\varepsilon} \circ S_{\varepsilon} \mathbb{1}_{\Sigma^{\varepsilon} \times S}(x, y)=\psi\left(\varepsilon y_{1}, \varepsilon\left[x_{2} / \varepsilon+1 / 2\right]+\varepsilon y_{2}, x_{3}, y\right) \mathbb{1}_{\Sigma^{\varepsilon} \times S}(x, y)
$$

is uniformly bounded in $\Sigma \times S$ and converges uniformly to $\psi$ on $\Sigma^{\varepsilon_{0}} \times S$ for each fixed $\varepsilon_{0}>0$, therefore, since $\mathcal{H}^{2}\left(\Sigma \backslash \Sigma^{\varepsilon}\right) \rightarrow 0$,

$$
\psi_{\varepsilon} \circ S_{\varepsilon} \mathbb{1}_{\Sigma^{\varepsilon} \times S} \rightarrow \psi \quad \text { strongly in } \quad L^{p}(\Sigma \times S), \quad \forall p \in[1,+\infty[.
$$

Assertion (i) (resp. (ii)) follows from (30) and (33) (resp. (30), (31) and (33)). Moreover, the combination of (30), (31) and (33) shows that

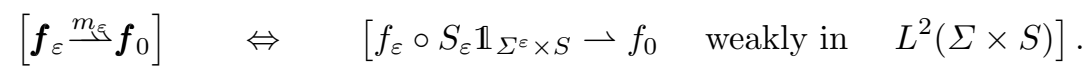

In order to prove the assertions (iii) we note at first that

$$
\boldsymbol{v}_{\varepsilon}\left(\boldsymbol{f}_{\varepsilon}\right)(x) \mathbb{1}_{T_{\varepsilon}}(x)=\left(\frac{1}{|S|} \int_{S} \boldsymbol{f}_{\varepsilon}\left(\varepsilon y_{1}, \varepsilon\left[x_{2} / \varepsilon+1 / 2\right]+\varepsilon y_{2}, x_{3}\right) d y\right) \mathbb{1}_{T_{\varepsilon}}(x),
$$

and we deduce from (30) and (32) that

$$
\int \boldsymbol{v}_{\varepsilon}\left(\boldsymbol{f}_{\varepsilon}\right)(x) \cdot \boldsymbol{\psi}\left(x, \frac{y_{\varepsilon}\left(x^{\prime}\right)}{\varepsilon}\right) d m_{\varepsilon}=\frac{1}{|S|} \int_{\Sigma^{\varepsilon} \times S} \boldsymbol{f}_{\varepsilon}\left(\varepsilon y_{1},\left[x_{2} / \varepsilon+1 / 2\right]+\varepsilon y_{2}, x_{3}\right) \widetilde{\boldsymbol{\psi}}_{\varepsilon}(x) d \mathcal{H}^{2}(x) d y
$$

where $\widetilde{\boldsymbol{\psi}}_{\varepsilon}(x):=\frac{1}{|S|} \int_{S} \boldsymbol{\psi}\left(\varepsilon y_{1}, \varepsilon\left[x_{2} / \varepsilon+1 / 2\right]+\varepsilon y_{2}, x_{3}, y\right) d y$. By the uniform continuity of $\boldsymbol{\psi}$, we have

$$
\begin{aligned}
& \left|\widetilde{\boldsymbol{\psi}}_{\varepsilon}(x)-\overline{\boldsymbol{\psi}}\left(\varepsilon y_{1},\left[x_{2} / \varepsilon+1 / 2\right]+\varepsilon y_{2}, x_{3}\right)\right| \mathbb{1}_{\Sigma^{\varepsilon} \times S} \leq C \varepsilon, \\
& \overline{\boldsymbol{\psi}}(x):=\frac{1}{|S|} \int_{S} \boldsymbol{\psi}(x, y) d \mathcal{H}^{2}(y) .
\end{aligned}
$$

Testing the two-scale convergence with respect to $\left(m_{\varepsilon}\right)$ of $\left(\boldsymbol{f}_{\varepsilon}\right)$ to $\boldsymbol{f}_{0}$ with the test function $\overline{\boldsymbol{\psi}}(x)$, taking (34) into account, we infer from (35) and (36) that

$$
\begin{aligned}
& \lim _{\varepsilon \rightarrow 0} \int \boldsymbol{v}_{\varepsilon}\left(\boldsymbol{f}_{\varepsilon}\right)(x) \cdot \boldsymbol{\psi}\left(x, \frac{y_{\varepsilon}\left(x^{\prime}\right)}{\varepsilon}\right) d m_{\varepsilon} \\
& =\lim _{\varepsilon \rightarrow 0} \frac{1}{|S|} \int_{\Sigma^{\varepsilon} \times S} \boldsymbol{f}_{\varepsilon}\left(\varepsilon y_{1},\left[x_{2} / \varepsilon+1 / 2\right]+\varepsilon y_{2}, x_{3}\right) \cdot \overline{\boldsymbol{\psi}}\left(\varepsilon y_{1},\left[x_{2} / \varepsilon+1 / 2\right]+\varepsilon y_{2}, x_{3}\right) d y d \mathcal{H}^{2}(x) \\
& =\frac{1}{|S|} \int_{\Sigma \times S} \boldsymbol{f}_{0}(x, y) \cdot \overline{\boldsymbol{\psi}}(x) d \mathcal{H}^{2}(x) d y=\frac{1}{|S|} \int_{\Sigma \times S}\left(\frac{1}{|S|} \int_{\Sigma \times S} \boldsymbol{f}_{0}(x, y) d \mathcal{H}^{2}(y)\right) \cdot \boldsymbol{\psi}(x, y) d \mathcal{H}^{2}(x) d y .
\end{aligned}
$$

Assertion (iii) a) is proved. For each $\left(x_{2}, x_{3}\right) \in \Sigma$ the mapping $x_{1} \rightarrow \boldsymbol{v}_{\varepsilon}\left(\boldsymbol{f}_{\varepsilon}\right)\left(x_{1}, x_{2}, x_{3}\right)$ is constant on the set $(-\varepsilon, \varepsilon) \times\left(x_{2}, x_{3}\right)$, and so in particular equal to $\mathfrak{v}_{\varepsilon}\left(\boldsymbol{f}_{\varepsilon}\right)\left(0, x_{2}, x_{3}\right)$. Hence the field $\mathfrak{v}_{\varepsilon}\left(\boldsymbol{f}_{\varepsilon}\right)\left(0, x_{2}, x_{3}\right)$ is a well defined element of $L^{2}\left(\Sigma ; \mathbb{R}^{k}\right)$ which will also be denoted by $\boldsymbol{\gamma}_{\Sigma}\left(\boldsymbol{v}_{\varepsilon}\left(\boldsymbol{f}_{\varepsilon}\right)\right)$, using for simplicity the same notation as for the trace operator from $H^{1}\left(\Omega ; \mathbb{R}^{k}\right)$ to $L^{2}\left(\Sigma ; \mathbb{R}^{k}\right)$. One can easily check that $\int_{\Sigma}\left|\boldsymbol{\gamma}_{\Sigma}\left(\boldsymbol{v}_{\varepsilon}\left(\boldsymbol{f}_{\varepsilon}\right)\right)\right|^{2} d \mathcal{H}^{2}=\int\left|\boldsymbol{v}_{\varepsilon}\left(\boldsymbol{f}_{\varepsilon}\right)\right|^{2} d m_{\varepsilon} \leq \int\left|\boldsymbol{f}_{\varepsilon}\right|^{2} d m_{\varepsilon}$, hence $\boldsymbol{\gamma}_{\Sigma}\left(\boldsymbol{v}_{\varepsilon}\left(\boldsymbol{f}_{\varepsilon}\right)\right)$ is bounded in $L^{2}\left(\Sigma ; \mathbb{R}^{k}\right)$. Given $\boldsymbol{\varphi} \in C\left(\bar{\Omega} ; \mathbb{R}^{k}\right)$, a straightforward computation yields

$$
\int_{\Sigma} \gamma_{\Sigma}\left(\mathfrak{v}_{\varepsilon}\left(f_{\varepsilon}\right)\right) \cdot \boldsymbol{\varphi}\left(0, x_{2}, x_{3}\right) d \mathcal{H}^{2}=\int \boldsymbol{f}_{\varepsilon} \cdot \widehat{\varphi}_{\varepsilon} d m_{\varepsilon}, \quad \widehat{\varphi}_{\varepsilon}(x):=\sum_{i \in I_{\varepsilon}}\left(f_{\varepsilon i-\frac{\varepsilon}{2}}^{\varepsilon i+\frac{\varepsilon}{2}} \varphi\left(0, t, x_{3}\right) d t\right) \mathbb{1}_{S_{\varepsilon}^{i}}\left(x^{\prime}\right) .
$$

Noticing that, by the uniform continuity of $\varphi$ on $\bar{\Omega}$, there holds $\sup _{x \in T_{\varepsilon}}\left|\boldsymbol{\varphi}(x)-\widehat{\varphi}_{\varepsilon}(x)\right| \leq C \varepsilon$, we deduce from the two-scale convergence with respect to $\left(m_{\varepsilon}\right)$ of $\left(\boldsymbol{f}_{\varepsilon}\right)$ to $\boldsymbol{f}_{0}$ that

$$
\begin{aligned}
\lim _{\varepsilon \rightarrow 0} \int_{\Sigma} \gamma_{\Sigma}\left(\boldsymbol{v}_{\varepsilon}\left(\boldsymbol{f}_{\varepsilon}\right)\right) \boldsymbol{\varphi}(x) d \mathcal{H}^{2} & =\lim _{\varepsilon \rightarrow 0} \int \boldsymbol{f}_{\varepsilon} \cdot \boldsymbol{\varphi} d m_{\varepsilon}=\frac{1}{|S|} \int_{\Sigma \times S} \boldsymbol{f}_{0}(x, y) \boldsymbol{\varphi}(x) d \mathcal{H}^{2}(x) d y \\
& =\int_{\Sigma}\left(f_{S} \boldsymbol{f}_{0}(x, y) d y\right) \cdot \boldsymbol{\varphi} d \mathcal{H}^{2} .
\end{aligned}
$$


Assertion (iii) b) is proved. The proof of Assertion (iii) c) is straightforward.

The next proposition states a lower semicontinuity property related to the two-scale convergence with respect to $\left(m_{\varepsilon}\right)$ when dealing with convex integrands.

Proposition 2 Let $\left(\boldsymbol{f}_{\varepsilon}\right)$ be a sequence in $L^{2}\left(\Omega ; \mathbb{R}^{k}\right)$ such that $\left(\boldsymbol{f}_{\varepsilon}\right)$ two-scale converges to $\boldsymbol{f}_{0}$ with respect to $\left(m_{\varepsilon}\right)$, and let $j: \mathbb{R}^{k} \rightarrow \mathbb{R}$ be a convex function. Then

$$
\liminf _{\varepsilon \rightarrow 0} \int j\left(\boldsymbol{f}_{\varepsilon}\right) d m_{\varepsilon} \geq \frac{1}{|S|} \int_{\Sigma \times S} j\left(\boldsymbol{f}_{0}\right) d \mathcal{H}^{2} \otimes d \mathcal{L}^{2} .
$$

Proof. Let us fix $\boldsymbol{\psi} \in C\left(\overline{\Omega \times S} ; \mathbb{R}^{k}\right)$. Denoting by $j^{\star}$ the Fenchel transform of $j$, by applying Fenchel inequality and then $(28)$ to the function $j^{\star}(\psi) \in C(\overline{\Omega \times S})$, we get

$$
\begin{aligned}
\liminf _{\varepsilon \rightarrow 0} \int j\left(\boldsymbol{f}_{\varepsilon}\right) d m_{\varepsilon}(x) & \geq \lim _{\varepsilon \rightarrow 0} \int \boldsymbol{f}_{\varepsilon} \boldsymbol{\psi}\left(x, \frac{y_{\varepsilon}\left(x^{\prime}\right)}{\varepsilon}\right)-j^{\star}\left(\boldsymbol{\psi}\left(x, \frac{y_{\varepsilon}\left(x^{\prime}\right)}{\varepsilon}\right)\right) d m_{\varepsilon}(x) \\
& =\frac{1}{|S|} \int_{\Sigma \times S} \boldsymbol{f}_{0} \boldsymbol{\psi}-j^{\star}(\boldsymbol{\psi}) d \mathcal{H}^{2}(x) d y .
\end{aligned}
$$

Taking the supremum of the right-hand side of (40) when $\psi$ varies in $C\left(\overline{\Omega \times S} ; \mathbb{R}^{k}\right)$, using a classical localization argument and the convexity assumption on $j$, we get

$$
\begin{aligned}
\liminf _{\varepsilon \rightarrow 0} \int j\left(\boldsymbol{f}_{\varepsilon}\right) d m_{\varepsilon}(x) & \geq \sup _{\boldsymbol{\psi} \in C\left(\overline{\Omega \times S} ; \mathbb{R}^{k}\right)} \frac{1}{|S|} \int_{\Sigma \times S} \boldsymbol{f}_{0} \boldsymbol{\psi}-j^{\star}(\boldsymbol{\psi}) d \mathcal{H}^{2}(x) d y \\
& =\frac{1}{|S|} \int_{\Sigma \times S} \sup _{\boldsymbol{\psi} \in \mathbb{R}^{k}}\left\{\boldsymbol{f}_{0} \boldsymbol{\psi}-j^{\star}(\boldsymbol{\psi})\right\} d \mathcal{H}^{2}(x) d y \\
& =\frac{1}{|S|} \int_{\Sigma \times S} j^{\star \star}\left(\boldsymbol{f}_{0}\right) d \mathcal{H}^{2}(x) d y=\frac{1}{|S|} \int_{\Sigma \times S} j\left(\boldsymbol{f}_{0}\right) d \mathcal{H}^{2}(x) d y
\end{aligned}
$$

The estimates established in the next lemma will be employed to study the link between the two-scale limit with respect to $\left(m_{\varepsilon}\right)$ of a bounded sequence in $H^{1}\left(\Omega ; \mathbb{R}^{k}\right)$ and the strong limit of its traces in $L^{2}\left(\Sigma ; \mathbb{R}^{k}\right)$. Also, they will take a crucial part in the proof of the apriori estimates established in Proposition 4 . In what follows, for each $\varphi \in H^{1}\left(\Omega ; \mathbb{R}^{3}\right)$, we denote by $\widehat{\varphi}$ and $\bar{\varphi}_{\varepsilon}$ the elements of $H^{1}\left(\Omega ; \mathbb{R}^{3}\right)$ defined by

$$
\begin{aligned}
& \overline{\boldsymbol{\varphi}}_{\varepsilon}(x):=f_{\left(-\frac{\varepsilon}{2}, \frac{\varepsilon}{2}\right)} \varphi\left(s_{1}, x_{2}, x_{3}\right) d s_{1}, \\
& \widehat{\varphi}(x):=\varphi\left(0, x_{2}, x_{3}\right) .
\end{aligned}
$$

Lemma 1 There exists a constant $C$ such that, for all $\varphi \in H^{1}\left(\Omega ; \mathbb{R}^{3}\right)$, see (3),

$$
\begin{aligned}
& \int_{\Sigma_{\varepsilon}}\left|\gamma_{\Sigma}\left(\mathfrak{v}_{\varepsilon}(\boldsymbol{\varphi})\right)-\gamma_{\Sigma}(\boldsymbol{\varphi})\right|^{2} d \mathcal{H}^{2} \leq C \varepsilon \int_{-\varepsilon / 2}^{\varepsilon / 2} \int_{\Sigma}|\nabla \boldsymbol{\varphi}|^{2} d x \\
& \int\left|\boldsymbol{\varphi}-\mathfrak{v}_{\varepsilon}(\boldsymbol{\varphi})\right|^{2} d m_{\varepsilon} \leq C \varepsilon^{2} \int|\nabla \boldsymbol{\varphi}|^{2} d m_{\varepsilon} \\
& \int|\boldsymbol{\varphi}-\hat{\boldsymbol{\varphi}}|^{2} d m_{\varepsilon} \leq C \varepsilon \int_{\Sigma_{\varepsilon}}|\nabla \boldsymbol{\varphi}|^{2} d x
\end{aligned}
$$

and

$$
\begin{aligned}
& \int|\widehat{\varphi}|^{2} d m_{\varepsilon} \leq C \int_{\Sigma}\left|\gamma_{\Sigma}(\boldsymbol{\varphi})\right|^{2} d \mathcal{H}^{2} \\
& \int_{\Sigma}\left|\frac{\partial \gamma_{\Sigma}\left(\mathfrak{v}_{\varepsilon 3}(\boldsymbol{\varphi})\right)}{\partial x_{3}}\right|^{2} d \mathcal{H}^{2} \leq C \int|\boldsymbol{e}(\boldsymbol{\varphi})|^{2} d m_{\varepsilon}
\end{aligned}
$$


Moreover for all $\varphi \in L^{2}\left(\Omega ; \mathbb{R}^{3}\right)$ and $k \in\{1,2,3\}$

$$
\int_{\Sigma}\left|\gamma_{\Sigma}\left(\mathfrak{v}_{\varepsilon k}(\boldsymbol{\varphi})\right)\right|^{2} d \mathcal{H}^{2}=\int\left|\mathfrak{v}_{\varepsilon k}(\boldsymbol{\varphi})\right|^{2} d m_{\varepsilon}
$$

Proof. We use the following estimate, whose proof is postponed to the end:

$$
\int_{\frac{-1}{2}}^{\frac{1}{2}}\left|f_{S} \boldsymbol{\psi} d s-\boldsymbol{\psi}\left(0, y_{2}\right)\right|^{2} d y_{2} \leq C \int_{Y}|\nabla \boldsymbol{\psi}|^{2} d y_{1} d y_{2} \quad \forall \boldsymbol{\psi} \in H^{1}\left(Y ; \mathbb{R}^{3}\right)
$$

By making suitable changes of variables in (45), we infer that for all $i \in I_{\varepsilon}$, there holds

$$
\int_{\varepsilon(i-1 / 2)}^{\varepsilon(i+1 / 2)}\left|f_{S_{\varepsilon}^{i}} \boldsymbol{\psi} d s-\boldsymbol{\psi}\left(0, x_{2}\right)\right|^{2} d x_{2} \leq C \varepsilon \int_{Y_{\varepsilon}^{i}}|\nabla \boldsymbol{\psi}|^{2} d x_{1} d x_{2} \quad \forall \boldsymbol{\psi} \in H^{1}\left(Y_{\varepsilon}^{i} ; \mathbb{R}^{3}\right) .
$$

Fixing $\varphi \in H^{1}\left(\Omega ; \mathbb{R}^{3}\right)$ and applying (46) for a.e. $x_{3} \in(-L, L)$ to $\boldsymbol{\psi}\left(x_{1}, x_{2}\right):=\varphi\left(x_{1}, x_{2}, x_{3}\right)$, then integrating with respect to $x_{3}$ over $(-L, L)$ and summing over $i \in I_{\varepsilon}$, we infer

$$
\int_{\Sigma_{\varepsilon}}\left|\gamma_{\Sigma}\left(\mathfrak{v}_{\varepsilon}(\varphi)\right)-\gamma_{\Sigma}(\varphi)\right|^{2} d \mathcal{H}^{2} \leq C \varepsilon \int_{-\varepsilon / 2}^{\varepsilon / 2} \int_{\Sigma}|\nabla \varphi|^{2} d x
$$

The proof of the first line of (42) is achieved.

Let us prove the second line of (42). By making suitable changes of variables in the classical PoincaréWirtinger inequality

$$
\int_{S}\left|\boldsymbol{\psi}-f_{S} \boldsymbol{\psi}\right|^{2} d y \leq C \int_{S}|\nabla \boldsymbol{\psi}|^{2} d y \quad \forall \boldsymbol{\psi} \in H^{1}\left(S ; \mathbb{R}^{2}\right)
$$

we infer

$$
\int_{S_{\varepsilon}^{i}}\left|\psi-f_{S_{\varepsilon}^{i}} \boldsymbol{\psi}\right|^{2} d x^{\prime} \leq C \varepsilon^{2} \int_{S_{\varepsilon}^{i}}|\nabla \psi|^{2} d x^{\prime} \quad \forall i \in I_{\varepsilon}, \forall \psi \in H^{1}\left(S_{\varepsilon}^{i} ; \mathbb{R}^{2}\right)
$$

By applying (47) for a.e. $x_{3} \in(-L, L)$ to $\psi\left(x_{1}, x_{2}\right)=\varphi\left(x_{1}, x_{2}, x_{3}\right)$, integrating with respect to $x_{3}$ over $(-L, L)$ and summing with respect to $i$ over $I_{\varepsilon}$, we deduce the second line of (42).

By Jensen's inequality we have

$$
\begin{aligned}
\int|\boldsymbol{\varphi}-\widehat{\varphi}|^{2} d m_{\varepsilon} & \leq \frac{C}{\varepsilon} \int_{\Sigma_{\varepsilon}}|\boldsymbol{\varphi}-\widehat{\varphi}|^{2} d x \leq \frac{C}{\varepsilon} \int_{\left(-\frac{\varepsilon}{2}, \frac{\varepsilon}{2}\right)} d x_{1} \int_{\Sigma}\left|\varphi\left(x_{1}, x_{2}, x_{3}\right)-\varphi\left(0, x_{2}, x_{3}\right)\right|^{2} d x_{2} d x_{3} \\
& \leq \int_{\Sigma}\left|\int_{\left(-\frac{\varepsilon}{2}, \frac{\varepsilon}{2}\right)}\right| \frac{\partial \varphi}{\partial x_{1}}\left(t_{1}, x_{2}, x_{3}\right)\left|d t_{1}\right|^{2} d x_{2} d x_{3} \\
& \leq C \varepsilon \int_{\Sigma_{\varepsilon}}|\nabla \varphi|^{2} d x
\end{aligned}
$$

which proves the third line of (42). 
By (25) and Jensen's inequality, there holds

$$
\begin{aligned}
\int_{\Sigma}\left|\frac{\partial \gamma_{\Sigma}\left(\mathfrak{v}_{\varepsilon 3}(\varphi)\right)}{\partial x_{3}}\right|^{2} d \mathcal{H}^{2} & =\sum_{i \in I_{\varepsilon}} \int_{-L}^{L} d x_{3} \int_{\varepsilon\left(i-\frac{1}{2}\right)}^{\varepsilon\left(i+\frac{1}{2}\right)} d x_{2}\left|f_{S_{\varepsilon}^{i}} \frac{\partial \varphi_{3}}{\partial x_{3}}\left(s_{1}, s_{2}, x_{3}\right) d s_{1} d s_{2}\right|^{2} \\
& \leq \sum_{i \in I_{\varepsilon}} \int_{-L}^{L} d x_{3} \int_{\varepsilon\left(i-\frac{1}{2}\right)}^{\varepsilon\left(i+\frac{1}{2}\right)} d x_{2} f_{S_{\varepsilon}^{i}}\left|\frac{\partial \varphi_{3}}{\partial x_{3}}\right|^{2}\left(s_{1}, s_{2}, x_{3}\right) d s_{1} d s_{2} \\
& =\varepsilon \sum_{i \in I_{\varepsilon}} \int_{-L}^{L} d x_{3} f_{S_{\varepsilon}^{i}}\left|\frac{\partial \varphi_{3}}{\partial x_{3}}\right|^{2}\left(s_{1}, s_{2}, x_{3}\right) d s_{1} d s_{2} \\
& =\frac{1}{\varepsilon|S|} \int_{T_{\varepsilon}}\left|\frac{\partial \varphi_{3}}{\partial x_{3}}\right|^{2} d x \leq C \int|\boldsymbol{e}(\boldsymbol{\varphi})|^{2} d m_{\varepsilon},
\end{aligned}
$$

yielding the second line of (43). Moreover, we have

$$
\int|\widehat{\varphi}|^{2} d m_{\varepsilon}=\frac{1}{\varepsilon|S|} \int_{T_{\varepsilon}}\left|\boldsymbol{\varphi}\left(0, x_{2}, x_{3}\right)\right|^{2} d x \leq \frac{1}{\varepsilon|S|} \int_{\left(-\frac{\varepsilon}{2}, \frac{\varepsilon}{2}\right) \times \Sigma}\left|\boldsymbol{\varphi}\left(0, x_{2}, x_{3}\right)\right|^{2} d x=\frac{1}{|S|} \int_{\Sigma}\left|\gamma_{\Sigma}(\boldsymbol{\varphi})\right|^{2} d \mathcal{H}^{2}
$$

The estimates (43) are proved. The estimate (44) results from the next computation, holding for $k \in$ $\{1,2,3\}$ :

$$
\begin{aligned}
\int_{\Sigma}\left|\gamma_{\Sigma}\left(\mathfrak{v}_{\varepsilon k}(\boldsymbol{\varphi})\right)\right|^{2} d \mathcal{H}^{2} & =\sum_{i \in I_{\varepsilon}} \int_{-L}^{L} d x_{3} \int_{\varepsilon\left(i-\frac{1}{2}\right)}^{\varepsilon\left(i+\frac{1}{2}\right)} d x_{2}\left|f_{S_{\varepsilon}^{i}} \varphi_{k}\left(s, x_{3}\right) d \mathcal{H}^{2}(s)\right|^{2} \\
& =\sum_{i \in I_{\varepsilon}} \int_{-L}^{L} d x_{3} \varepsilon\left|f_{S_{\varepsilon}^{i}} \varphi_{k}\left(s, x_{3}\right) d \mathcal{H}^{2}(s)\right|^{2} \\
& =\sum_{i \in I_{\varepsilon}} \int_{-L}^{L} d x_{3} \varepsilon \frac{1}{\left|S_{\varepsilon}^{i}\right|} \int_{S_{\varepsilon}^{i}}\left|f_{S_{\varepsilon}^{i}} \varphi_{k}\left(s, x_{3}\right) d \mathcal{H}^{2}(s)\right|^{2} d x_{1} d x_{2} \\
& =\frac{1}{\varepsilon|S|} \int_{T_{\varepsilon}}\left|\mathfrak{v}_{\varepsilon k}(\boldsymbol{\varphi})\right|^{2} d x=\int\left|\mathfrak{v}_{\varepsilon k}(\boldsymbol{\varphi})\right|^{2} d m_{\varepsilon} .
\end{aligned}
$$

Proof of (45). If the first line of (45) is not satisfied, there exists $\left(\boldsymbol{\psi}_{n}\right) \subset H^{1}\left(Y ; \mathbb{R}^{3}\right)$ such that

$$
\int_{\left(\frac{-1}{2}, \frac{1}{2}\right)}\left|f_{S} \boldsymbol{\psi}_{n} d s-\boldsymbol{\psi}_{n}\left(0, y_{2}\right)\right|^{2} d y_{2}=1, \quad \int_{Y}\left|\nabla \boldsymbol{\psi}_{n}\right|^{2} d y_{1} d y_{2} \leq \frac{1}{n}
$$

After possibly substracting a constant vector to $\psi_{n}$, we can assume that

$$
f_{S} \psi_{n} d y=0
$$

From the Poincaré-Wirtinger inequality

$$
\int_{Y}\left|\boldsymbol{\psi}-f_{S} \boldsymbol{\psi} d s\right|^{2} d y \leq C \int_{Y}|\nabla \boldsymbol{\psi}|^{2} d y \quad \forall \boldsymbol{\psi} \in H^{1}\left(Y ; \mathbb{R}^{3}\right)
$$

we deduce that $\left(\boldsymbol{\psi}_{n}\right)$ is bounded in $H^{1}\left(Y ; \mathbb{R}^{3}\right)$ and weakly converges, thanks to (50) and up to a subsequence (still denoted by $\psi_{n}$ ), to 0 . By the continuity of the trace application from $H^{1}\left(Y ; \mathbb{R}^{3}\right)$ weak to $L^{2}\left(\{0\} \times\left(\frac{-1}{2}, \frac{1}{2}\right) ; \mathbb{R}^{3}\right)$ strong, the trace $\boldsymbol{\psi}_{n}\left(0, y_{2}\right)$ converges strongly to 0 in $L^{2}\left(\{0\} \times\left(\frac{-1}{2}, \frac{1}{2}\right) ; \mathbb{R}^{3}\right)$. This is in contradiction with the fact that by (49) and (50), the norm in $L^{2}\left(\{0\} \times\left(\frac{-1}{2}, \frac{1}{2}\right) ; \mathbb{R}^{3}\right)$ of $\boldsymbol{\psi}_{n}\left(0, y_{2}\right)$ is equal to 1 .

We are now in a position to investigate the link between the two-scale limit with respect to $\left(m_{\varepsilon}\right)$ of a bounded sequence in $H^{1}\left(\Omega ; \mathbb{R}^{k}\right)$ and the strong limit of its traces in $L^{2}\left(\Sigma ; \mathbb{R}^{k}\right)$. 
Proposition 3 Assume that $\left(\boldsymbol{f}_{\varepsilon}\right)$ weakly converges in $H^{1}\left(\Omega ; \mathbb{R}^{k}\right)$ to some $\boldsymbol{f} \in H^{1}\left(\Omega ; \mathbb{R}^{k}\right)$. Then, $\left(\boldsymbol{f}_{\varepsilon}\right)$ two-scale converges with respect to $\left(m_{\varepsilon}\right)$ to the field $\boldsymbol{f}_{0} \in L^{2}\left(\Sigma \times S ; \mathbb{R}^{k}\right)$ defined by

$$
\boldsymbol{f}_{0}(x, y):=\gamma_{\Sigma}(\boldsymbol{f})(x) \quad \text { for } \mathcal{H}^{2} \otimes \mathcal{L}^{2} \text {-a.e. } \quad(x, y) \in \Sigma \times S .
$$

In addition, the sequence $\left(\boldsymbol{v}_{\varepsilon}\left(\boldsymbol{f}_{\varepsilon}\right)\right)$ defined by (25) two-scale converges with respect to $\left(m_{\varepsilon}\right)$ to $\boldsymbol{f}_{0}$. Furthermore, the sequence $\left(\boldsymbol{\gamma}_{\Sigma}\left(\mathbf{v}_{\varepsilon}\left(\boldsymbol{f}_{\varepsilon}\right)\right)\right)$ strongly converges in $L^{2}\left(\Sigma ; \mathbb{R}^{k}\right)$ to $\gamma_{\Sigma}(\boldsymbol{f})$.

Proof. By the first line of (42), we have

$$
\begin{aligned}
& \int_{\Sigma}\left|\gamma_{\Sigma}\left(\mathfrak{v}_{\varepsilon}\left(\boldsymbol{f}_{\varepsilon}\right)\right)-\gamma_{\Sigma}(\boldsymbol{f})\right|^{2} d \mathcal{H}^{2} \\
& \leq C \int_{\Sigma_{\varepsilon}}\left|\gamma_{\Sigma}\left(\mathfrak{v}_{\varepsilon}\left(\boldsymbol{f}_{\varepsilon}\right)\right)-\gamma_{\Sigma}\left(\boldsymbol{f}_{\varepsilon}\right)\right|^{2} d \mathcal{H}^{2}+C \int_{\Sigma_{\varepsilon}}\left|\boldsymbol{\gamma}_{\Sigma}\left(\boldsymbol{f}_{\varepsilon}\right)-\gamma_{\Sigma}(\boldsymbol{f})\right|^{2} d \mathcal{H}^{2}+C \int_{\Sigma \backslash \Sigma_{\varepsilon}}\left|\gamma_{\Sigma}(\boldsymbol{f})\right|^{2} d \mathcal{H}^{2} \\
& \leq C \varepsilon \int_{-\varepsilon / 2}^{\varepsilon / 2} \int_{\Sigma}\left|\nabla \boldsymbol{f}_{\varepsilon}\right|^{2} d x+C \int_{\Sigma}\left|\gamma_{\Sigma}\left(\boldsymbol{f}_{\varepsilon}\right)-\gamma_{\Sigma}(\boldsymbol{f})\right|^{2} d \mathcal{H}^{2}+C \int_{\Sigma \backslash \Sigma_{\varepsilon}}\left|\gamma_{\Sigma}(\boldsymbol{f})\right|^{2} d \mathcal{H}^{2} .
\end{aligned}
$$

Since $\left(\boldsymbol{f}_{\varepsilon}\right)$ weakly converges to $\boldsymbol{f}$ in $H^{1}\left(\Omega ; \mathbb{R}^{k}\right)$, the sequence $\left(\boldsymbol{\gamma}_{\Sigma}\left(\boldsymbol{f}_{\varepsilon}\right)\right)$ strongly converges to $\boldsymbol{\gamma}_{\Sigma}(\boldsymbol{f})$ in $L^{2}\left(\Sigma ; \mathbb{R}^{k}\right)$. Since the sequence $\left(\left|\boldsymbol{\gamma}_{\Sigma}(\boldsymbol{f})\right|^{2} \mathbb{1}_{\Sigma \backslash \Sigma^{\varepsilon}}\right)$ is dominated by $\left|\boldsymbol{\gamma}_{\Sigma}(\boldsymbol{f})\right|^{2} \in L^{1}(\Sigma)$ and $\mathcal{H}^{2}$-a.e. converges to 0 on $\Sigma$, by the Dominated Convergence Theorem, there holds: $\lim _{\varepsilon \rightarrow 0} \int_{\Sigma \backslash \Sigma^{\varepsilon}}\left|\gamma_{\Sigma}(f)\right|^{2} d \mathcal{H}^{2}=0$. It then follows from (52) that the sequence $\left(\gamma_{\Sigma}\left(\boldsymbol{v}_{\varepsilon}\left(\boldsymbol{f}_{\varepsilon}\right)\right)\right)$ strongly converges in $L^{2}\left(\Sigma ; \mathbb{R}^{k}\right)$ to $\gamma_{\Sigma}(\boldsymbol{f})$. Given $\psi \in C\left(\overline{\Omega \times S} ; \mathbb{R}^{k}\right)$, we consider the fields $\overline{\boldsymbol{\psi}}_{\varepsilon}^{\Sigma} \in L^{\infty}\left(\Sigma ; \mathbb{R}^{k}\right)$ and $\overline{\boldsymbol{\psi}}^{\Sigma} \in C\left(\bar{\Sigma} ; \mathbb{R}^{k}\right)$ defined by

$$
\begin{aligned}
& \overline{\boldsymbol{\psi}}_{\varepsilon}^{\Sigma}\left(x_{2}, x_{3}\right):=\sum_{i \in I_{\varepsilon}}\left(f_{S_{\varepsilon}^{i}} \psi\left(s_{1}, s_{2}, x_{3}, \frac{y_{\varepsilon}(s)}{\varepsilon}\right) d s_{1} d s_{2}\right) \mathbb{1}_{\left(\varepsilon i-\frac{\varepsilon}{2}, \varepsilon i+\frac{\varepsilon}{2}\right)}\left(x_{2}\right), \\
& \bar{\psi}^{\Sigma}\left(x_{2}, x_{3}\right):=f_{S} \boldsymbol{\psi}\left(0, x_{2}, x_{3}, y\right) d y .
\end{aligned}
$$

By the change of variables formula, there holds (see (26))

$$
\overline{\boldsymbol{\psi}}^{\Sigma}\left(x_{2}, x_{3}\right)=f_{S_{\varepsilon}^{i}} \boldsymbol{\psi}\left(0, x_{2}, x_{3}, \frac{y_{\varepsilon}(s)}{\varepsilon}\right) d s \quad \forall i \in I_{\varepsilon} .
$$

Therefore, for all $\left(x_{2}, x_{3}\right) \in\left(\varepsilon i-\frac{\varepsilon}{2}, \varepsilon i+\frac{\varepsilon}{2}\right) \times(-L, L)$ we have

$$
\left|\overline{\boldsymbol{\psi}}^{\Sigma}\left(x_{2}, x_{3}\right)-\overline{\boldsymbol{\psi}}_{\varepsilon}^{\Sigma}\left(x_{2}, x_{3}\right)\right| \leq \sup _{(s, y) \in S_{\varepsilon}^{i} \times S}\left|\boldsymbol{\psi}\left(0, x_{2}, x_{3}, y\right)-\boldsymbol{\psi}\left(s, x_{3}, y\right)\right| .
$$

By (53) we have

$$
\left\|\overline{\boldsymbol{\psi}}_{\varepsilon}^{\Sigma}\right\|_{L^{\infty}\left(\Sigma ; \mathbb{R}^{k}\right)} \leq\|\boldsymbol{\psi}\|_{L^{\infty}\left(\Omega \times S ; \mathbb{R}^{k}\right)}
$$

hence the sequence $\left(\bar{\psi}_{\varepsilon}^{\Sigma}\right)$ is bounded in $L^{\infty}\left(\Sigma ; \mathbb{R}^{k}\right)$. On the other hand, one can check by using the uniform continuity of $\boldsymbol{\psi}$ on $\overline{\Omega \times S}$, that the sequence $\left(\overline{\boldsymbol{\psi}}_{\varepsilon}^{\Sigma}\right)$ uniformly converges to $\overline{\boldsymbol{\psi}}^{\Sigma}$ on each compact subset of $\Sigma$. We deduce that

By (25) and (53) there holds

$$
\overline{\boldsymbol{\psi}}_{\varepsilon}^{\Sigma} \rightarrow \overline{\boldsymbol{\psi}}^{\Sigma} \quad \text { strongly in } L^{q}\left(\Sigma ; \mathbb{R}^{k}\right) \quad \forall q \in[1,+\infty) .
$$

$$
\begin{aligned}
\int \mathfrak{v}_{\varepsilon}\left(\boldsymbol{f}_{\varepsilon}\right) \cdot \boldsymbol{\psi}\left(x, \frac{y_{\varepsilon}\left(x^{\prime}\right)}{\varepsilon}\right) & d m_{\varepsilon}=\frac{1}{\varepsilon|S|} \sum_{i \in I_{\varepsilon}} \int_{-L}^{L} d x_{3} \int_{S_{\varepsilon}^{i}}\left(f_{S_{\varepsilon}^{i}} \boldsymbol{f}_{\varepsilon}\left(s_{1}, s_{2}, x_{3}\right) d s\right) \cdot \boldsymbol{\psi}\left(x, \frac{y_{\varepsilon}\left(x^{\prime}\right)}{\varepsilon}\right) d x_{1} d x_{2} \\
& =\frac{1}{\varepsilon|S|} \sum_{i \in I_{\varepsilon}} \int_{-L}^{L} d x_{3}\left(f_{S_{\varepsilon}^{i}} \boldsymbol{f}_{\varepsilon}\left(s_{1}, s_{2}, x_{3}\right) d s\right) \cdot \int_{S_{\varepsilon}^{i}} \boldsymbol{\psi}\left(s_{1}, s_{2}, x_{3}, \frac{y_{\varepsilon}(s)}{\varepsilon}\right) d s \\
& =\sum_{i \in I_{\varepsilon}} \int_{\left(\varepsilon i-\frac{\varepsilon}{2}, \varepsilon i+\frac{\varepsilon}{2}\right) \times(-L, L)} \gamma_{\Sigma}\left(\mathbf{v}_{\varepsilon}\left(\boldsymbol{f}_{\varepsilon}\right)\right) \cdot \overline{\boldsymbol{\psi}}_{\varepsilon}^{\Sigma} d \mathcal{H}^{2}=\int_{\Sigma} \boldsymbol{\gamma}_{\Sigma}\left(\mathfrak{v}_{\varepsilon}\left(\boldsymbol{f}_{\varepsilon}\right)\right) \cdot \overline{\boldsymbol{\psi}}_{\varepsilon}^{\Sigma} d \mathcal{H}^{2} .
\end{aligned}
$$


By passing to the limit as $\varepsilon \rightarrow 0$, thanks to the strong convergences of $\left(\boldsymbol{\gamma}_{\Sigma}\left(\boldsymbol{v}_{\varepsilon}\left(\boldsymbol{f}_{\varepsilon}\right)\right)\right)$ to $\gamma_{\Sigma}(\boldsymbol{f})$ in $L^{2}\left(\Sigma ; \mathbb{R}^{k}\right)$ and of $\left(\overline{\boldsymbol{\psi}}_{\varepsilon}^{\Sigma}\right)$ to $\overline{\boldsymbol{\psi}}^{\Sigma}$ in $L^{2}\left(\Sigma ; \mathbb{R}^{k}\right)$ (see $\left.(54)\right)$, we get

$$
\lim _{\varepsilon \rightarrow 0} \int \boldsymbol{v}_{\varepsilon}\left(\boldsymbol{f}_{\varepsilon}\right) \cdot \boldsymbol{\psi}\left(x, \frac{y_{\varepsilon}\left(x^{\prime}\right)}{\varepsilon}\right) d m_{\varepsilon}=\int_{\Sigma} \boldsymbol{\gamma}_{\Sigma}(\boldsymbol{f}) \cdot \overline{\boldsymbol{\psi}}^{\Sigma} d \mathcal{H}^{2} .
$$

On the other hand, by (53) there holds

$$
\int_{\Sigma} \boldsymbol{\gamma}_{\Sigma}(\boldsymbol{f}) \cdot \overline{\boldsymbol{\psi}}^{\Sigma} d \mathcal{H}^{2}=\frac{1}{|S|} \int_{\Sigma \times S} \boldsymbol{\gamma}_{\Sigma}(\boldsymbol{f})(x) \cdot \boldsymbol{\psi}(x, y) d \mathcal{H}^{2}(x) d y
$$

By the arbitrary choice of $\boldsymbol{\psi}$, we infer from (56) and (57) that the sequence $\left(\boldsymbol{v}_{\varepsilon}\left(\boldsymbol{f}_{\varepsilon}\right)\right)$ two-scale converges with respect to $\left(m_{\varepsilon}\right)$ to the field $\boldsymbol{f}_{0} \in L^{2}\left(\Sigma ; \mathbb{R}^{k}\right)$ defined by $(51)$.

The proof of the first statement is achieved provided we show that $\left(\boldsymbol{f}_{\varepsilon}\right)$ two-scale converges to $\boldsymbol{f}_{0}$ with respect to $\left(m_{\varepsilon}\right)$. To that aim, let us fix $\boldsymbol{\psi} \in C\left(\overline{\Omega \times S} ; \mathbb{R}^{k}\right)$. By $(25)$, Hölder's inequality and Jensen's inequality, we have

$$
\begin{aligned}
\left|\int\left(\boldsymbol{f}_{\varepsilon}-\boldsymbol{v}_{\varepsilon}\left(\boldsymbol{f}_{\varepsilon}\right)\right) \cdot \boldsymbol{\psi}\left(x, \frac{y_{\varepsilon}\left(x^{\prime}\right)}{\varepsilon}\right) d m_{\varepsilon}\right|^{2} & \leq C \int\left|\boldsymbol{f}_{\varepsilon}-\boldsymbol{v}_{\varepsilon}\left(\boldsymbol{f}_{\varepsilon}\right)\right|^{2} d m_{\varepsilon} \\
& \leq C \frac{1}{\varepsilon|S|} \sum_{i \in I_{\varepsilon}} \int_{-L}^{L} d x_{3} \int_{S_{\varepsilon}^{i}}\left|\boldsymbol{f}_{\varepsilon}-f_{S_{\varepsilon}^{i}} \boldsymbol{f}_{\varepsilon}\left(s_{1}, s_{2}, x_{3}\right) d s\right|^{2} d x_{1} d x_{2} .
\end{aligned}
$$

By making suitable changes of variables in the following Poincaré-Wirtinger inequality

$$
\int_{S}\left|\boldsymbol{\varphi}-f_{S} \boldsymbol{\varphi} d s\right|^{2} d x \leq C \int_{S}|\nabla \varphi|^{2} d x \quad \forall \boldsymbol{\varphi} \in H^{1}\left(S ; \mathbb{R}^{k}\right)
$$

we deduce that for a. e. $x_{3} \in(-L, L)$, there holds

$$
\int_{S_{\varepsilon}^{i}}\left|\boldsymbol{f}_{\varepsilon}-f_{S_{\varepsilon}^{i}} \boldsymbol{f}_{\varepsilon}\left(s_{1}, s_{2}, x_{3}\right) d s\right|^{2} d x_{1} d x_{2} \leq C \varepsilon^{2} \int_{S_{\varepsilon}^{i}}\left|\nabla \boldsymbol{f}_{\varepsilon}\left(x_{1}, x_{2}, x_{3}\right)\right|^{2} d x_{1} d x_{2} .
$$

Joining (58) and (59), we infer

$$
\left|\int\left(\boldsymbol{f}_{\varepsilon}-\boldsymbol{v}_{\varepsilon}\left(\boldsymbol{f}_{\varepsilon}\right)\right) \cdot \boldsymbol{\psi}\left(x, \frac{y_{\varepsilon}\left(x^{\prime}\right)}{\varepsilon}\right) d m_{\varepsilon}\right|^{2} \leq C \varepsilon \int_{T_{\varepsilon}}\left|\nabla \boldsymbol{f}_{\varepsilon}\right|^{2} d x .
$$

We deduce from (56), (57) and (60) that

$$
\lim _{\varepsilon \rightarrow 0} \int \boldsymbol{f}_{\varepsilon} \cdot \boldsymbol{\psi}\left(x, \frac{y_{\varepsilon}\left(x^{\prime}\right)}{\varepsilon}\right) d m_{\varepsilon}=\frac{1}{|S|} \int_{\Sigma \times S} \boldsymbol{\gamma}_{\Sigma}(\boldsymbol{f})(x) \cdot \boldsymbol{\psi}(x, y) d \mathcal{H}^{2}(x) d y .
$$

Therefore, by the arbitrary choice of $\boldsymbol{\psi}$, the sequence $\left(\boldsymbol{f}_{\varepsilon}\right)$ two-scale converges with respect to $\left(m_{\varepsilon}\right)$ to $f_{0}$.

\section{A priori estimates, identification relations}

The following section is mainly devoted to the study of the asymptotic behaviour of the solution $\left(\boldsymbol{u}_{\varepsilon}\right)$ of (7) and of the sequences $\left(\boldsymbol{v}_{\varepsilon}\left(\boldsymbol{u}_{\varepsilon}\right) m_{\varepsilon}\right)$ and $\left(\boldsymbol{u}_{\varepsilon} m_{\varepsilon}\right)$. The main results of this section are stated in Proposition

4. Their proofs rely on some basic inequalities established in the previous section in Lemma 1 and also on those established in the next lemma. 
Lemma 2 The following estimates hold for all $i \in I_{\varepsilon}$ :

$$
\begin{array}{ll}
\int_{T_{\varepsilon}^{i}}\left|\varepsilon \varphi_{1}\right|^{2}+\left|\varepsilon \varphi_{2}\right|^{2}+\left|\varphi_{3}\right|^{2} d x \leq C \int_{T_{\varepsilon}^{i}}|\boldsymbol{e}(\boldsymbol{\varphi})|^{2} d x & \forall \boldsymbol{\varphi} \in H_{0}^{1}\left(\Omega ; \mathbb{R}^{3}\right), \\
\int_{T_{\varepsilon}^{i}}\left|\varepsilon \mathfrak{v}_{\varepsilon 1}(\boldsymbol{\varphi})\right|^{2}+\left|\varepsilon \mathfrak{v}_{\varepsilon 2}(\boldsymbol{\varphi})\right|^{2}+\left|\mathfrak{v}_{\varepsilon 3}(\boldsymbol{\varphi})\right|^{2} d x \leq C \int_{T_{\varepsilon}^{i}}|\boldsymbol{e}(\boldsymbol{\varphi})|^{2} d x & \forall \boldsymbol{\varphi} \in H_{0}^{1}\left(\Omega ; \mathbb{R}^{3}\right) .
\end{array}
$$

Proof. We consider the set

$$
V:=\left\{\boldsymbol{\psi} \in H^{1}\left(T, \mathbb{R}^{3}\right), \boldsymbol{\psi}=0 \text { on } S \times\{-L\}\right\} .
$$

The space $V$ is a closed linear subset of $H^{1}\left(T, \mathbb{R}^{3}\right)$ satisfying $V \cap \mathcal{R}=\{0\}$, where $\mathcal{R}$ denotes the space of rigid displacements, therefore by Korn inequality there holds

$$
\int_{T}|\boldsymbol{\psi}|^{2} d z \leq C \int_{T}|\boldsymbol{e}(\boldsymbol{\psi})|^{2} d z \quad \forall \boldsymbol{\psi} \in V .
$$

Let us fix $\boldsymbol{\varphi} \in H_{0}^{1}\left(\Omega ; \mathbb{R}^{3}\right)$. By applying (62) to the field $\boldsymbol{\psi}$ defined by $\psi_{\alpha}\left(z_{1}, z_{2}, z_{3}\right):=\varphi_{\alpha}\left(\varepsilon\left(z_{1}-\right.\right.$ $\left.\left.\left(\boldsymbol{y}_{\varepsilon}^{i}(z)\right)_{1}\right), \varepsilon\left(z_{2}-\left(\boldsymbol{y}_{\varepsilon}^{i}(z)\right)_{2}\right), z_{3}\right)$ for $\alpha \in\{1,2\}$ and $\psi_{3}(z):=\frac{1}{\varepsilon} \varphi_{3}\left(\varepsilon\left(z_{1}-\left(\boldsymbol{y}_{\varepsilon}^{i}(z)\right)_{1}\right), \varepsilon\left(z_{2}-\left(\boldsymbol{y}_{\varepsilon}^{i}(z)\right)_{2}\right), z_{3}\right)$, and then by making a suitable change of variable, we get

$$
\int_{T_{\varepsilon}^{i}}\left|\varepsilon \varphi_{1}\right|^{2}+\left|\varepsilon \varphi_{2}\right|^{2}+\left|\varphi_{3}\right|^{2} d x \leq C \int_{T_{\varepsilon}^{i}}|\boldsymbol{e}(\boldsymbol{\varphi})|^{2} d x
$$

On the other hand, it easily follows from (25), Fubini Theorem and Jensen's inequality that

$$
\int_{T_{\varepsilon}^{i}}\left|\varepsilon \mathfrak{v}_{\varepsilon 1}(\boldsymbol{\varphi})\right|^{2}+\left|\varepsilon \mathfrak{v}_{\varepsilon 2}(\boldsymbol{\varphi})\right|^{2}+\left|\mathfrak{v}_{\varepsilon 3}(\boldsymbol{\varphi})\right|^{2} d x \leq C \int_{T_{\varepsilon}^{i}}\left|\varepsilon \varphi_{1}\right|^{2}+\left|\varepsilon \varphi_{2}\right|^{2}+\left|\varphi_{3}\right|^{2} d x .
$$

The estimates (61) result from (63) and (64).

The next proposition specifies the asymptotic behavior of the solution $\boldsymbol{u}_{\varepsilon}$ of $(7)$ and of some associated auxiliary sequences. The strong relative compactness of $\left(\boldsymbol{u}_{\varepsilon}\right)$ in $H_{0}^{1}\left(\Omega ; \mathbb{R}^{3}\right)$ is established at the end of the paper in Section 5.2.

Proposition 4 Let $\boldsymbol{u}_{\varepsilon}$ be the solution of (7) and let $\boldsymbol{v}_{\varepsilon}\left(\boldsymbol{u}_{\varepsilon}\right)$ be defined by (25). Then:

(i) the next estimates hold true

$$
\begin{aligned}
& \left|\boldsymbol{u}_{\varepsilon}\right|_{H_{0}^{1}\left(\Omega ; \mathbb{R}^{3}\right)}^{2} \leq C, \quad \int\left|\boldsymbol{e}\left(\boldsymbol{u}_{\varepsilon}\right)\right|^{2} d m_{\varepsilon} \leq C \varepsilon^{m-1}, \quad \int\left\{\left|\boldsymbol{u}_{\varepsilon}\right|^{2}+\left|\mathfrak{v}_{\varepsilon}\left(\boldsymbol{u}_{\varepsilon}\right)\right|^{2}\right\} d m_{\varepsilon} \leq C, \\
& \int\left\{\left|u_{\varepsilon 1}\right|^{2}+\left|u_{\varepsilon 2}\right|^{2}+\left|\frac{u_{\varepsilon 3}}{\varepsilon}\right|^{2}+\left|\mathfrak{v}_{\varepsilon 1}\left(\boldsymbol{u}_{\varepsilon}\right)\right|^{2}+\left|\mathfrak{v}_{\varepsilon 2}\left(\boldsymbol{u}_{\varepsilon}\right)\right|^{2}+\left|\frac{\mathfrak{v}_{\varepsilon 3}\left(\boldsymbol{u}_{\varepsilon}\right)}{\varepsilon}\right|^{2}\right\} d m_{\varepsilon} \leq C \varepsilon^{m-3}, \\
& \int\left|\boldsymbol{u}_{\varepsilon}-\boldsymbol{v}_{\varepsilon}\left(\boldsymbol{u}_{\varepsilon}\right)\right|^{2} d m_{\varepsilon} \leq C \varepsilon, \quad \int_{\Sigma}\left|\boldsymbol{\gamma}_{\Sigma}\left(\boldsymbol{v}_{\varepsilon}\left(\boldsymbol{u}_{\varepsilon}\right)\right)\right|^{2} d \mathcal{H}^{2} \leq C .
\end{aligned}
$$

(ii) If $m \geq 1$, the next convergences take place, up to a subsequence, for some $\boldsymbol{u} \in H_{0}^{1}\left(\Omega ; \mathbb{R}^{3}\right), \boldsymbol{u}_{0}, \boldsymbol{v}_{0} \in$ $L^{2}\left(\Sigma \times S ; \mathbb{R}^{3}\right), \Xi_{0} \in L^{2}\left(\Sigma \times S ; \mathbb{S}^{3}\right)$ :

$$
\begin{aligned}
& \boldsymbol{u}_{\varepsilon} \rightarrow \boldsymbol{u} \quad \text { weakly in } H_{0}^{1}\left(\Omega ; \mathbb{R}^{3}\right) \text {, } \\
& \boldsymbol{\gamma}_{\Sigma}\left(\boldsymbol{u}_{\varepsilon}\right) \rightarrow \boldsymbol{\gamma}_{\Sigma}(\boldsymbol{u}), \quad \boldsymbol{\gamma}_{\Sigma}\left(\boldsymbol{v}_{\varepsilon}\left(\boldsymbol{u}_{\varepsilon}\right)\right) \rightarrow \boldsymbol{\gamma}_{\Sigma}(\boldsymbol{u}) \quad \text { strongly in } L^{2}\left(\Sigma ; \mathbb{R}^{3}\right),
\end{aligned}
$$

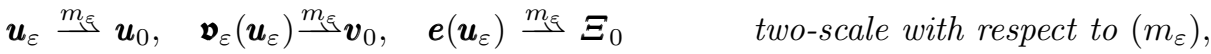

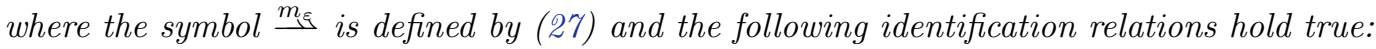

$$
\boldsymbol{v}_{0}(x, y)=\boldsymbol{u}_{0}(x, y)=\gamma_{\Sigma}(\boldsymbol{u})(x) \quad \text { for } \mathcal{H}^{2} \otimes \mathcal{L}^{2} \text {-a.e. }(x, y) \in \Sigma \times S,
$$




$$
\begin{gathered}
\frac{\partial \gamma_{\Sigma}\left(u_{3}\right)}{\partial x_{3}} \in L^{2}(\Sigma), \quad \gamma_{\Sigma}\left(u_{3}\right)=0 \quad \text { on } \quad\left\{x \in \Sigma, x_{3} \in\{-L, L\}\right\}, \\
\frac{\partial \gamma_{\Sigma}\left(u_{3}\right)}{\partial x_{3}}(x)=\left(\Xi_{0}\right)_{33}(x, y) \quad \text { for } \quad \mathcal{H}^{2} \otimes \mathcal{L}^{2} \text {-a.e. } \quad(x, y) \in \Sigma \times S, \\
\gamma_{\Sigma}\left(u_{3}\right)=0 \quad \text { if } \quad m>1 .
\end{gathered}
$$

(iii) If $m \geq 3$, then besides (66), the next convergences take place up to a subsequence, for some $w_{0} \in$ $L^{2}(\Sigma \times S), \Upsilon_{0} \in L^{2}\left(\Sigma \times S ; \mathbb{S}^{3}\right), w \in L^{2}(\Sigma)$ :

$$
\begin{aligned}
& \frac{u_{\varepsilon 3}}{\varepsilon} \frac{m_{\varepsilon}}{\varepsilon} w_{0}, \frac{1}{\varepsilon} \boldsymbol{e}\left(\boldsymbol{u}_{\varepsilon}\right) \stackrel{m_{\Sigma}}{\boldsymbol{\Upsilon}_{0}} \quad \text { two-scale with respect to }\left(m_{\varepsilon}\right), \\
& \frac{\gamma_{\Sigma}\left(\mathfrak{v}_{\varepsilon 3}\left(\boldsymbol{u}_{\varepsilon}\right)\right)}{\varepsilon} \rightarrow w \text { weakly in } L^{2}(\Sigma) .
\end{aligned}
$$

Moreover there holds

$$
\begin{aligned}
& \gamma_{\Sigma}\left(u_{3}\right)=0, \quad \frac{\partial^{2} \boldsymbol{\gamma}_{\Sigma}(\boldsymbol{u})}{\partial x_{3}^{2}} \in L^{2}\left(\Sigma ; \mathbb{R}^{3}\right), \quad \boldsymbol{\gamma}_{\Sigma}(\boldsymbol{u})=\frac{\partial \boldsymbol{\gamma}_{\Sigma}(\boldsymbol{u})}{\partial x_{3}}=0 \quad \text { on } \partial \Sigma \cap\left\{x \in \mathbb{R}^{3}, x_{3} \in\{-L, L\}\right\} \\
& w_{0}(x, y)=w(x)-\sum_{\alpha=1}^{2} \frac{\partial \gamma_{\Sigma}\left(u_{\alpha}\right)}{\partial x_{3}}(x) y_{\alpha}, \text { in } \Sigma \times S, \quad \frac{\partial w}{\partial x_{3}} \in L^{2}(\Sigma), \\
& \Upsilon_{033}=\frac{\partial w}{\partial x_{3}}-\frac{\partial^{2} \gamma_{\Sigma}\left(u_{1}\right)}{\partial x_{3}^{2}} y_{1}-\frac{\partial^{2} \gamma_{\Sigma}\left(u_{2}\right)}{\partial x_{3}^{2}} y_{2} .
\end{aligned}
$$

Furthermore,

$$
\gamma_{\Sigma}(\boldsymbol{u})=0 \text { if } m>3
$$

(iv) We have $\boldsymbol{u} \in \mathcal{D}_{m}$, where $\mathcal{D}_{m}$ is given by (9), (11), (16), or (13), in accordance with the choice of $m$.

Proof. (i) We multiply (7) by $\boldsymbol{u}_{\varepsilon}$, integrate by parts over $\Omega$, and use Cauchy Schwarz inequality to find

$$
\int_{\Omega} \boldsymbol{\sigma}_{\varepsilon}: \boldsymbol{e}\left(\boldsymbol{u}_{\varepsilon}\right) d x=\int_{\Omega} \boldsymbol{f} . \boldsymbol{u}_{\varepsilon} \leq\left(\int_{\Omega}|\boldsymbol{f}|^{2} d x\right)^{\frac{1}{2}}\left(\int_{\Omega}\left|\boldsymbol{u}_{\varepsilon}\right|^{2} d x\right)^{\frac{1}{2}} .
$$

By applying Poincaré and Korn inequalities in $H_{0}^{1}\left(\Omega ; \mathbb{R}^{3}\right)$, we get

$$
\begin{aligned}
\int_{\Omega}\left|\boldsymbol{u}_{\varepsilon}\right|^{2} d x & \leq C \int_{\Omega}\left|\boldsymbol{e}\left(\boldsymbol{u}_{\varepsilon}\right)\right|^{2} d x \leq C \int_{\Omega \backslash T_{\varepsilon}}\left|\boldsymbol{e}\left(\boldsymbol{u}_{\varepsilon}\right)\right|^{2} d x+\frac{C}{\varepsilon^{m}} \int_{T_{\varepsilon}}\left|\boldsymbol{e}\left(\boldsymbol{u}_{\varepsilon}\right)\right|^{2} d x \\
& \leq C \int_{\Omega} \boldsymbol{\sigma}_{\varepsilon}: \boldsymbol{e}\left(\boldsymbol{u}_{\varepsilon}\right) d x
\end{aligned}
$$

We infer from (73) and (74) that

$$
\left|\boldsymbol{u}_{\varepsilon}\right|_{H_{0}^{1}\left(\Omega ; \mathbb{R}^{3}\right)}^{2} \leq C, \quad \int\left|\boldsymbol{e}\left(\boldsymbol{u}_{\varepsilon}\right)\right|^{2} d m_{\varepsilon} \leq C \varepsilon^{m-1} .
$$

By the third line of (42), the first line of (43), (74), and the continuity of the trace application from $H^{1}\left(\Omega ; \mathbb{R}^{3}\right)$ to $L^{2}\left(\Omega ; \mathbb{R}^{3}\right)$, there holds

$$
\begin{aligned}
\int\left|\boldsymbol{u}_{\varepsilon}\right|^{2} d m_{\varepsilon} & \leq C \int\left|\boldsymbol{u}_{\varepsilon}-\widehat{\boldsymbol{u}}_{\varepsilon}\right|^{2} d m_{\varepsilon}+C \int\left|\widehat{\boldsymbol{u}}_{\varepsilon}\right|^{2} d m_{\varepsilon} \\
& \leq C \varepsilon \int_{\Sigma_{\varepsilon}}\left|\nabla \boldsymbol{u}_{\varepsilon}\right|^{2} d x+C \int_{\Sigma}\left|\gamma_{\Sigma}\left(\boldsymbol{u}_{\varepsilon}\right)\right|^{2} d \mathcal{H}^{2} \leq C\left|\boldsymbol{u}_{\varepsilon}\right|_{H^{1}\left(\Omega ; \mathbb{R}^{3}\right)}^{2} \leq C .
\end{aligned}
$$


We deduce from (61) and (75) that

$$
\int\left\{\left|u_{\varepsilon 1}\right|^{2}+\left|u_{\varepsilon 2}\right|^{2}+\left|\frac{u_{\varepsilon 3}}{\varepsilon}\right|^{2}\right\} d m_{\varepsilon} \leq \frac{C}{\varepsilon^{2}} \int\left|\boldsymbol{e}\left(\boldsymbol{u}_{\varepsilon}\right)\right|^{2} d m_{\varepsilon}=C \varepsilon^{m-3}
$$

and that

$$
\int\left\{\left|\mathfrak{v}_{\varepsilon 1}\left(\boldsymbol{u}_{\varepsilon}\right)\right|^{2}+\left|\mathfrak{v}_{\varepsilon 2}\left(\boldsymbol{u}_{\varepsilon}\right)\right|^{2}+\left|\frac{\mathfrak{v}_{\varepsilon 3}\left(\boldsymbol{u}_{\varepsilon}\right)}{\varepsilon}\right|^{2}\right\} d m_{\varepsilon} \leq \frac{C}{\varepsilon^{2}} \int\left|\boldsymbol{e}\left(\boldsymbol{u}_{\varepsilon}\right)\right|^{2} d m_{\varepsilon}=C \varepsilon^{m-3} .
$$

By (42) and (75), we have

$$
\int\left|\boldsymbol{u}_{\varepsilon}-\boldsymbol{v}_{\varepsilon}\left(\boldsymbol{u}_{\varepsilon}\right)\right|^{2} d m_{\varepsilon} \leq C \varepsilon \int_{-\varepsilon / 2}^{\varepsilon / 2} \int_{\Sigma}\left|\nabla \boldsymbol{u}_{\varepsilon}\right|^{2} d x \leq C \varepsilon\left|\boldsymbol{u}_{\varepsilon}\right|_{H_{0}^{1}\left(\Omega ; \mathbb{R}^{3}\right)}^{2} \leq C \varepsilon .
$$

By the first line of $(42),(74),(75),(79)$, and by the continuity of the trace application from $H^{1}\left(\Omega ; \mathbb{R}^{3}\right)$ to $L^{2}\left(\Omega ; \mathbb{R}^{3}\right)$, there holds

$$
\begin{aligned}
\int_{\Sigma}\left|\boldsymbol{\gamma}_{\Sigma}\left(\boldsymbol{v}_{\varepsilon}\left(\boldsymbol{u}_{\varepsilon}\right)\right)\right|^{2} d \mathcal{H}^{2} & =\int_{\Sigma^{\varepsilon}}\left|\boldsymbol{\gamma}_{\Sigma}\left(\boldsymbol{v}_{\varepsilon}\left(\boldsymbol{u}_{\varepsilon}\right)\right)\right|^{2} d \mathcal{H}^{2} \\
& \leq C \int_{\Sigma^{\varepsilon}}\left|\boldsymbol{\gamma}_{\Sigma}\left(\boldsymbol{u}_{\varepsilon}\right)-\gamma_{\Sigma}\left(\boldsymbol{v}_{\varepsilon}\left(\boldsymbol{u}_{\varepsilon}\right)\right)\right|^{2} d \mathcal{H}^{2}+C \int_{\Sigma}\left|\gamma_{\Sigma}\left(\boldsymbol{u}_{\varepsilon}\right)\right|^{2} d \mathcal{H}^{2} \\
& \leq C \varepsilon+C\left|\boldsymbol{u}_{\varepsilon}\right|_{H^{1}\left(\Omega ; \mathbb{R}^{3}\right)}^{2} \leq C .
\end{aligned}
$$

Collecting (75)-(80), the estimates (65) are proved.

(ii) By $(65)$, the sequence $\left(\boldsymbol{u}_{\varepsilon}\right)$ is bounded in $H_{0}^{1}\left(\Omega ; \mathbb{R}^{3}\right)$ hence weakly converges, up to a subsequence, to some $\boldsymbol{u} \in H_{0}^{1}\left(\Omega ; \mathbb{R}^{3}\right)$. From the compactness of the trace operator from $H_{0}^{1}\left(\Omega ; \mathbb{R}^{3}\right)$ to $L^{2}\left(\Sigma ; \mathbb{R}^{3}\right)$, we deduce that the sequence $\left(\gamma_{\Sigma}\left(\boldsymbol{u}_{\varepsilon}\right)\right)$ strongly converges to $\gamma_{\Sigma}(\boldsymbol{u})$ in $L^{2}\left(\Sigma ; \mathbb{R}^{3}\right)$, and from Proposition 3 that the sequence $\left(\gamma_{\Sigma}\left(\boldsymbol{v}_{\varepsilon}\left(\boldsymbol{u}_{\varepsilon}\right)\right)\right)$ strongly converges to $\gamma_{\Sigma}(\boldsymbol{u})$ in $L^{2}\left(\Sigma ; \mathbb{R}^{3}\right)$. The convergences stated up to a subsequence in the last line of (66) result from Proposition 1 (ii) and from the fact that by (65), the estimate $\sup _{\varepsilon>0} \int\left|\boldsymbol{f}_{\varepsilon}\right|^{2} d m_{\varepsilon}<+\infty$ takes place for $\boldsymbol{f}_{\varepsilon} \in\left\{\boldsymbol{u}_{\varepsilon}, \boldsymbol{v}_{\varepsilon}\left(\boldsymbol{u}_{\varepsilon}\right), \boldsymbol{e}\left(\boldsymbol{u}_{\varepsilon}\right)\right\}$. Assertion (67) is a consequence of (66) and Proposition 3. Let us fix $\psi \in C^{\infty}(\bar{\Omega} ; \mathcal{D}(S))$. Since $u_{\varepsilon} \in H_{0}^{1}\left(\Omega ; \mathbb{R}^{3}\right)$, we have

$$
\int \frac{\partial u_{\varepsilon 3}}{\partial x_{3}} \psi\left(x, \frac{y_{\varepsilon}\left(x^{\prime}\right)}{\varepsilon}\right) d m_{\varepsilon}=-\int u_{\varepsilon 3} \frac{\partial \psi}{\partial x_{3}}\left(x, \frac{y_{\varepsilon}\left(x^{\prime}\right)}{\varepsilon}\right) d m_{\varepsilon} .
$$

By passing to the limit as $\varepsilon \rightarrow 0$, thanks to the two-scale convergences with respect to $\left(m_{\varepsilon}\right)$ of $\left(\boldsymbol{e}\left(\boldsymbol{u}_{\varepsilon}\right)\right)$ to $\boldsymbol{\Xi}_{0}$ and of $\left(\boldsymbol{u}_{\varepsilon}\right)$ to $\boldsymbol{\gamma}_{\Sigma}(\boldsymbol{u})$ (see $(66),(67)$ ), we infer

$$
\frac{1}{|S|} \int_{\Sigma \times S}\left(\Xi_{0}\right)_{33}(x, y) \psi(x, y) d \mathcal{H}^{2}(x) d y=-\frac{1}{|S|} \int_{\Sigma \times S} \gamma_{\Sigma}\left(u_{3}\right)(x) \frac{\partial \psi}{\partial x_{3}}(x, y) d \mathcal{H}^{2}(x) d y
$$

Since this equality is satisfied in particular for all $\psi \in \mathcal{D}(\Omega \times S)$, we deduce

$$
\frac{\partial \gamma_{\Sigma}\left(u_{3}\right)}{\partial x_{3}}(x)=\left(\Xi_{0}\right)_{33}(x, y) \quad \text { for } \quad \mathcal{H}^{2} \otimes \mathcal{L}^{2} \text {-a.e. }(x, y) \in \Sigma \times S
$$

As $\Xi_{0} \in L^{2}\left(\Sigma \times S ; \mathbb{S}^{3}\right)$, we infer that $\frac{\partial \gamma_{\Sigma}\left(u_{3}\right)}{\partial x_{3}} \in L^{2}(\Sigma)$. Choosing then $\psi \in C^{\infty}(\bar{\Omega} ; \mathcal{D}(S))$ and integrating (81) by parts with respect to $x_{3}$, taking (82) into account, we obtain

$$
\int_{\left\{x \in \Sigma, x_{3} \in\{-L, L\}\right\} \times S} \gamma_{\Sigma}\left(u_{3}\right)(x) \psi(x, y) d \mathcal{H}^{1}(x) d y=0,
$$

yielding, by the arbitrary choice of $\psi$,

$$
\gamma_{\Sigma}\left(u_{3}\right)=0 \text { on }\left\{x \in \Sigma, x_{3} \in\{-L, L\}\right\} .
$$

Assertion (68) is proved. Assertion (69) follows from the second line of (65) and the second line of (66).

(iii) If $m \geq 3$, then by (65) we have 


$$
\sup _{\varepsilon>0} \int\left|\frac{\mathfrak{v}_{\varepsilon 3}\left(\boldsymbol{u}_{\varepsilon}\right)}{\varepsilon}\right|^{2} d m_{\varepsilon}<+\infty, \quad \sup _{\varepsilon>0} \int\left|\frac{u_{\varepsilon 3}}{\varepsilon}\right|^{2} d m_{\varepsilon}<+\infty, \quad \sup _{\varepsilon>0} \int\left|\frac{1}{\varepsilon} \boldsymbol{e}\left(\boldsymbol{u}_{\varepsilon}\right)\right|^{2} d m_{\varepsilon}<+\infty .
$$

The convergences stated, up to a subsequence, in the first line of (70), then follow from Proposition 1 (ii). As regards those stated in the second line of (70), they result from the fact that by (44) and by the second line of (65), there holds

$$
\int_{\Sigma}\left|\frac{\gamma_{\Sigma}\left(\mathfrak{v}_{\varepsilon 3}\left(\boldsymbol{u}_{\varepsilon}\right)\right)}{\varepsilon}\right|^{2} d \mathcal{H}^{2} \leq C\left(\int\left|\frac{\mathfrak{v}_{\varepsilon 3}\left(\boldsymbol{u}_{\varepsilon}\right)}{\varepsilon}\right|^{2} d m_{\varepsilon}\right) \leq C .
$$

We deduce from the two-scale convergence of $\left(\frac{u_{\varepsilon 3}}{\varepsilon}\right)$ to $w_{0}$ with respect to $\left(m_{\varepsilon}\right)$ that $\left(u_{\varepsilon 3}\right)$ two-scale converges to 0 with respect to $\left(m_{\varepsilon}\right)$. It follows then from Proposition 3 that

$$
\gamma_{\Sigma}\left(u_{3}\right)=0
$$

To prove (71), we fix a test field $\boldsymbol{\Psi} \in C^{\infty}\left(\bar{\Omega}, \mathcal{D}\left(S ; \mathbb{S}^{3}\right)\right)$ such that $\Psi_{\alpha \beta}=0$ for all $(\alpha, \beta) \in\{1,2\}^{2}$. By integration by parts, we have:

$$
\begin{aligned}
\varepsilon \int_{T_{\varepsilon}} \frac{1}{\varepsilon} \boldsymbol{e}\left(\boldsymbol{u}_{\varepsilon}\right)(x): \boldsymbol{\Psi}\left(x, \frac{y_{\varepsilon}\left(x^{\prime}\right)}{\varepsilon}\right) d m_{\varepsilon}=\sum_{i=1}^{3}-\varepsilon \int_{T_{\varepsilon}} \frac{u_{\varepsilon 3}(x)}{\varepsilon} \frac{\partial \Psi_{3 i}}{\partial x_{i}}\left(x, \frac{y_{\varepsilon}\left(x^{\prime}\right)}{\varepsilon}\right) d m_{\varepsilon} \\
\quad-\sum_{\alpha=1}^{2} \int_{T_{\varepsilon}} u_{\varepsilon \alpha}(x) \frac{\partial \Psi_{\alpha 3}}{\partial x_{3}}\left(x, \frac{y_{\varepsilon}\left(x^{\prime}\right)}{\varepsilon}\right) d m_{\varepsilon}-\sum_{\alpha=1}^{2} \int_{T_{\varepsilon}} \frac{u_{\varepsilon 3}(x)}{\varepsilon} \frac{\partial \Psi_{3 \alpha}}{\partial y_{\alpha}}\left(x, \frac{y_{\varepsilon}\left(x^{\prime}\right)}{\varepsilon}\right) d m_{\varepsilon} .
\end{aligned}
$$

By passing to the limit as $\varepsilon \rightarrow 0$, thanks to the two-scale convergences stated in (70), we obtain

$$
\sum_{\alpha=1}^{2}-\iint_{\Sigma \times S} \gamma_{\Sigma}\left(u_{\alpha}\right)(x) \frac{\partial \Psi_{\alpha 3}}{\partial x_{3}}(x, y) d \mathcal{H}^{2}(x) d y-\iint_{\Sigma \times S} w_{0}(x, y) \frac{\partial \Psi_{3 \alpha}}{\partial y_{\alpha}}(x, y) d \mathcal{H}^{2}(x) d y=0
$$

Fixing $\alpha \in\{1,2\}$ and choosing $\boldsymbol{\Psi}$ such that $\Psi_{i j}=0$ if $\{i, j\} \neq\{\alpha, 3\}$, we get

$$
-\iint_{\Sigma \times S} \gamma_{\Sigma}\left(u_{\alpha}\right)(x) \frac{\partial \Psi_{\alpha 3}}{\partial x_{3}}(x, y) d \mathcal{H}^{2}(x) d y-\iint_{\Sigma \times S} w_{0}(x, y) \frac{\partial \Psi_{3 \alpha}}{\partial y_{\alpha}}(x, y) d \mathcal{H}^{2}(x) d y=0
$$

Choosing at first arbitrary fields $\Psi_{\alpha 3}$ in $\mathcal{D}(\Omega ; \mathcal{D}(S))$, we deduce that

$$
\frac{\partial w_{0}}{\partial y_{\alpha}}(x, y)=-\frac{\partial \gamma_{\Sigma}\left(u_{\alpha}\right)}{\partial x_{3}}(x) \quad \text { in } \mathcal{D}^{\prime}(\Sigma \times S)
$$

hence $w_{0}$, as a distribution on $\Sigma \times S$, can be written for a suitable $c \in \mathcal{D}^{\prime}(\Sigma)$ under the following form:

$$
w_{0}(x, y)=-y_{1} \frac{\partial \gamma_{\Sigma}\left(u_{1}\right)}{\partial x_{3}}(x)-y_{2} \frac{\partial \gamma_{\Sigma}\left(u_{2}\right)}{\partial x_{3}}(x)+c(x)
$$

As $w_{0}$ belongs to $L^{2}(\Sigma \times S)$, we infer

$$
c \in L^{2}(\Sigma), \quad \frac{\partial \gamma_{\Sigma}\left(u_{\alpha}\right)}{\partial x_{3}} \in L^{2}(\Sigma) \quad(\alpha \in\{1,2\})
$$

and (87) holds a.e. on $\Sigma \times S$. We then deduce from Proposition 1 (iii) b), (70) and (88) that

$$
w(x)=f_{S} w_{0}(x, y) d y=c(x)
$$


Next, we multiply (84) by $\frac{1}{\varepsilon}$ and choose a field $\boldsymbol{\Psi}$ such that $\Psi_{i j}=0$ if $(i, j) \neq(3,3)$. Passing to the limit as $\varepsilon \rightarrow 0$, taking into account the two-scale convergences with respect to $\left(m_{\varepsilon}\right)$ of $\frac{1}{\varepsilon} \boldsymbol{e}\left(\boldsymbol{u}_{\varepsilon}\right)$ to $\boldsymbol{\Upsilon}_{0}$ and of $\frac{u_{\varepsilon 3}}{\varepsilon}$ to $w_{0}$ given by $(88)$ and $(89)$, we obtain

$$
\begin{aligned}
\iint_{\Sigma \times S} & \Upsilon_{033}(x, y) \Psi_{33}(x, y) d \mathcal{H}^{2}(x) d y \\
& =-\iint_{\Sigma \times S}\left(-y_{1} \frac{\partial \gamma_{\Sigma}\left(u_{1}\right)}{\partial x_{3}}(x)-y_{2} \frac{\partial \gamma_{\Sigma}\left(u_{2}\right)}{\partial x_{3}}(x)+w(x)\right) \frac{\partial \Psi_{33}}{\partial x_{3}}(x, y) d \mathcal{H}^{2}(x) d y
\end{aligned}
$$

Choosing at first an arbitrary $\Psi_{33} \in \mathcal{D}(\Omega ; \mathcal{D}(S))$, we conclude that

$$
\begin{aligned}
& \frac{\partial w}{\partial x_{3}} \in L^{2}(\Sigma), \quad \frac{\partial^{2} \gamma_{\Sigma}(\boldsymbol{u})}{\partial x_{3}^{2}} \in L^{2}\left(\Sigma ; \mathbb{R}^{3}\right), \\
& \left(\boldsymbol{\Upsilon}_{0}\right)_{33}=\frac{\partial w}{\partial x_{3}}(x)-\sum_{\alpha=1}^{2} \frac{\partial^{2} \gamma_{\Sigma}\left(u_{\alpha}\right)}{\partial x_{3}^{2}}(x) y_{\alpha}, \quad \text { in } \Sigma \times S,
\end{aligned}
$$

then, choosing an arbitrary $\Psi_{33} \in C^{\infty}(\bar{\Omega} ; \mathcal{D}(S))$ in $(90)$ and integrating by parts, we obtain

$$
0=\iint_{\partial \Sigma \cap\left\{x \in \mathbb{R}^{3}, x_{3} \in\{-L, L\}\right\} \times S}\left(-y_{1} \frac{\partial \gamma_{\Sigma}\left(u_{1}\right)}{\partial x_{3}}(x)-y_{2} \frac{\partial \gamma_{\Sigma}\left(u_{2}\right)}{\partial x_{3}}(x)+w(x)\right) \Psi_{33}(x, y) d \mathcal{H}^{1}(x) d y,
$$

yielding

$$
\begin{aligned}
& w=0 \text { on } \partial \Sigma \cap\left\{x \in \mathbb{R}^{3}, x_{3} \in\{-L, L\}\right\} \\
& \frac{\partial \boldsymbol{\gamma}_{\Sigma}(\boldsymbol{u})}{\partial x_{3}}=0 \text { on } \partial \Sigma \cap\left\{x \in \mathbb{R}^{3}, x_{3} \in\{-L, L\}\right\}
\end{aligned}
$$

Assertion (71) is proved.

Finally, if $m>3$, then by the second line of (65) the sequence $\left(\int\left|\boldsymbol{u}_{\varepsilon}\right|^{2} d m_{\varepsilon}\right)$ converges to 0 . We deduce from Proposition 2 (applied to $j():.=|.|^{2}$ ) that $\boldsymbol{u}_{0}=0$. The assertion (72) then results from (67).

(iv) Assertion (iv) follows from (66), (71), and (72).

\section{Proof of Theorem 1}

\subsection{Weak convergence}

Let us briefly outline the proof of Theorem 1. In the spirit of Tartar's method [34], we will multiply (7) by an appropriate sequence of oscillating test fields $\left(\phi_{\varepsilon}\right)$ and, by passing to the limit as $\varepsilon \rightarrow 0$ in accordance with the convergences established in proposition 4 , obtain a variational formulation of the limit problem satisfied by $\boldsymbol{u}$. The test field $\boldsymbol{\phi}_{\varepsilon}$ is constructed in terms of some fixed $\boldsymbol{\phi} \in \mathcal{D}\left(\Omega ; \mathbb{R}^{3}\right)$ such that $\boldsymbol{\phi} \in \mathcal{D}_{m}$ (see (9), (11), (16), (18)). We fix a smooth domain $S^{\prime}$ of $\mathbb{R}^{2}$ such that

$$
\bar{S} \subset S^{\prime} \subset \overline{S^{\prime}} \subset Y,
$$

and a function $\varrho \in \mathcal{D}(Y)$ such that

$$
\varrho=0 \quad \text { in } Y \backslash S^{\prime}, \quad \varrho=1 \quad \text { in } S, \quad 0 \leq \varrho \leq 1 .
$$

The function $\varrho_{\varepsilon}$ defined on $\bar{\Omega}$ by (see $(26)$ )

$$
\varrho_{\varepsilon}(x):=\sum_{i \in I_{\varepsilon}} \varrho\left(\frac{y_{\varepsilon}\left(x^{\prime}\right)}{\varepsilon}\right) \mathbb{1}_{Y_{\varepsilon}^{i}}\left(x^{\prime}\right),
$$

satisfies

$$
\varrho_{\varepsilon} \in C^{\infty}(\bar{\Omega}), \quad \varrho_{\varepsilon}=0 \quad \text { in } \Omega \backslash T_{\varepsilon}^{\prime}, \quad \varrho_{\varepsilon}=1 \quad \text { in } T_{\varepsilon}, \quad\left|\nabla \varrho_{\varepsilon}\right| \leq \frac{C}{\varepsilon},
$$


where $T_{\varepsilon}^{\prime}$ is defined by substituting $S^{\prime}$ for $S$ in (2). The test field will then be defined by

$$
\boldsymbol{\phi}_{\varepsilon}:=\left(1-\varrho_{\varepsilon}\right) \boldsymbol{\phi}+\varrho_{\varepsilon} \boldsymbol{\chi}_{\varepsilon},
$$

in terms of $\chi_{\varepsilon}$ given by (102), (110) (assuming $\phi_{3}=0$ if $1<m<3$ ), or (120), depending on the choice of $m$.

We multiply equation (7) by $\phi_{\varepsilon}$ and integrate it by parts over $\Omega$. We get (see (19)):

$$
a_{\varepsilon, m}\left(\boldsymbol{u}_{\varepsilon}, \boldsymbol{\phi}_{\varepsilon}\right)=\int_{\Omega} \boldsymbol{f} \cdot \boldsymbol{\phi}_{\varepsilon} d x
$$

It is easy to check that

$$
\lim _{\varepsilon \rightarrow 0} \int_{\Omega} f . \phi_{\varepsilon} d x=\int_{\Omega} f . \phi d x .
$$

In order to compute the limit of the left hand side of (97), we split it into a sum of three terms:

$$
\begin{aligned}
& a_{\varepsilon, m}\left(\boldsymbol{u}_{\varepsilon}, \phi_{\varepsilon}\right)=I_{1 \varepsilon}+I_{2 \varepsilon}+I_{3 \varepsilon} ; \quad I_{1 \varepsilon}=\int_{\Omega \backslash T_{\varepsilon}^{\prime}} \frac{E}{1+\nu}\left(\frac{\nu}{1-2 \nu} \operatorname{tr}\left(\boldsymbol{e}\left(\boldsymbol{u}_{\varepsilon}\right)\right) \boldsymbol{I}+\boldsymbol{e}\left(\boldsymbol{u}_{\varepsilon}\right)\right): \boldsymbol{e}(\boldsymbol{\phi}) d x, \\
& I_{2 \varepsilon}=\int_{T_{\varepsilon}^{\prime} \backslash T_{\varepsilon}} \frac{E}{1+\nu}\left(\frac{\nu}{1-2 \nu} \operatorname{tr}\left(\boldsymbol{e}\left(\boldsymbol{u}_{\varepsilon}\right)\right) \boldsymbol{I}+\boldsymbol{e}\left(\boldsymbol{u}_{\varepsilon}\right)\right): \boldsymbol{e}\left(\boldsymbol{\phi}_{\varepsilon}\right) d x, \\
& I_{3 \varepsilon}=\frac{|S|}{\varepsilon^{m-1}} \int \frac{E_{T}}{1+\nu}\left(\frac{\nu}{1-2 \nu} \operatorname{tr}\left(\boldsymbol{e}\left(\boldsymbol{u}_{\varepsilon}\right)\right) \boldsymbol{I}+\boldsymbol{e}\left(\boldsymbol{u}_{\varepsilon}\right)\right): \boldsymbol{e}\left(\boldsymbol{\chi}_{\varepsilon}\right) d m_{\varepsilon} .
\end{aligned}
$$

After possibly extracting a subsequence, we can assume that the convergences stated in Proposition 4 take place. In particular, the sequence $\frac{E}{1+\nu}\left(\frac{\nu}{1-2 \nu} \operatorname{tr}\left(\boldsymbol{e}\left(\boldsymbol{u}_{\varepsilon}\right)\right) \boldsymbol{I}+\boldsymbol{e}\left(\boldsymbol{u}_{\varepsilon}\right)\right)$ converges weakly to $\frac{E}{1+\nu}\left(\frac{\nu}{1-2 \nu} \operatorname{tr}(\boldsymbol{e}(\boldsymbol{u})) \boldsymbol{I}+\boldsymbol{e}(\boldsymbol{u})\right)$ in $L^{2}\left(\Omega, \mathbb{S}^{3}\right)$. On the other hand, since $\left|T_{\varepsilon}^{\prime}\right| \rightarrow 0$, the sequence $\boldsymbol{e}(\boldsymbol{\phi}) \mathbf{1}_{\Omega \backslash T_{\varepsilon}^{\prime}}$ converges strongly in $L^{2}\left(\Omega, \mathbb{S}^{3}\right)$ to $\boldsymbol{e}(\boldsymbol{\phi})$. We infer

$$
\lim _{\varepsilon \rightarrow 0} I_{1 \varepsilon}=a_{0}(\boldsymbol{u}, \boldsymbol{\phi})=\int_{\Omega} \frac{E}{1+\nu}\left(\frac{\nu}{1-2 \nu} \operatorname{tr}(\boldsymbol{e}(\boldsymbol{u})) \boldsymbol{I}+\boldsymbol{e}(\boldsymbol{u})\right): \boldsymbol{e}(\boldsymbol{\phi}) d x .
$$

The $\boldsymbol{\chi}_{\varepsilon}$ will be chosen such that

$$
\left|\boldsymbol{\phi}-\boldsymbol{\chi}_{\varepsilon}\right|_{L^{\infty}\left(T_{\varepsilon}^{\prime}\right)} \leq C \varepsilon, \quad\left|\boldsymbol{e}\left(\boldsymbol{\phi}-\boldsymbol{\chi}_{\varepsilon}\right)\right|_{L^{\infty}\left(T_{\varepsilon}^{\prime}\right)}\left|\boldsymbol{e}\left(\boldsymbol{\phi}_{\varepsilon}\right)\right| \leq C,
$$

(see (102), (110), (120)) therefore by (95) and (96) we have

$$
\left|\boldsymbol{e}\left(\boldsymbol{\phi}_{\varepsilon}\right)\right| \leq C \quad \text { in } T_{\varepsilon}^{\prime} \backslash T_{\varepsilon},
$$

hence by Cauchy-Schwartz inequality, there holds

$$
\begin{aligned}
\limsup _{\varepsilon \rightarrow 0} I_{2 \varepsilon} & \leq \limsup _{\varepsilon \rightarrow 0} \int_{T_{\varepsilon}^{\prime} \backslash T_{\varepsilon}} \frac{E}{1+\nu}\left(\frac{\nu}{1-2 \nu} \operatorname{tr}\left(\boldsymbol{e}\left(\boldsymbol{u}_{\varepsilon}\right)\right) \boldsymbol{I}+\boldsymbol{e}\left(\boldsymbol{u}_{\varepsilon}\right)\right): \boldsymbol{e}\left(\boldsymbol{\phi}_{\varepsilon}\right) d x \\
& \leq \limsup _{\varepsilon \rightarrow 0} C\left|T_{\varepsilon}^{\prime} \backslash T_{\varepsilon}\right|^{\frac{1}{2}}\left(\int_{T_{\varepsilon}^{\prime} \backslash T_{\varepsilon}}\left|\frac{E}{1+\nu}\left(\frac{\nu}{1-2 \nu} \operatorname{tr}\left(\boldsymbol{e}\left(\boldsymbol{u}_{\varepsilon}\right)\right) \boldsymbol{I}+\boldsymbol{e}\left(\boldsymbol{u}_{\varepsilon}\right)\right)\right|^{2} d x\right)^{\frac{1}{2}} \\
& \leq C \limsup _{\varepsilon \rightarrow 0} C\left|T_{\varepsilon}^{\prime} \backslash T_{\varepsilon}\right|^{\frac{1}{2}}=0 .
\end{aligned}
$$

We distinguish then different cases.

Case $m=1$. We set (see (25) and Remark 2 below)

$$
\boldsymbol{\chi}_{\varepsilon}:=\mathfrak{v}_{\varepsilon}(\boldsymbol{\phi})-\varepsilon\left(\begin{array}{c}
0 \\
0 \\
\frac{\partial \mathfrak{v}_{\varepsilon 1}(\boldsymbol{\phi})}{\partial x_{3}} \frac{y_{\varepsilon 1}\left(x^{\prime}\right)}{\varepsilon}+\frac{\partial \mathfrak{v}_{\varepsilon 2}(\boldsymbol{\phi})}{\partial x_{3}} \frac{y_{\varepsilon 2}\left(x^{\prime}\right)}{\varepsilon}
\end{array}\right)+\varepsilon \boldsymbol{q}\left(x, \frac{y_{\varepsilon}\left(x^{\prime}\right)}{\varepsilon}\right),
$$


where $\boldsymbol{y}_{\varepsilon}(x)$ is given by $(26)$ and

$$
\boldsymbol{q}(x, y):=\left(\begin{array}{c}
-\nu_{1} \frac{\partial \mathfrak{v}_{\varepsilon 3}(\boldsymbol{\phi})}{\partial x_{3}} y_{1} \\
-\nu_{1} \frac{\partial \mathfrak{v}_{\varepsilon 3}(\boldsymbol{\phi})}{\partial x_{3}} y_{2} \\
0
\end{array}\right)
$$

By (25) and (103) there holds in $T_{\varepsilon}$ :

$$
\boldsymbol{e}\left(\boldsymbol{\chi}_{\varepsilon}\right)=\frac{\partial \mathfrak{v}_{\varepsilon 3}(\boldsymbol{\phi})}{\partial x_{3}}\left(\begin{array}{ccc}
-\nu_{1} & 0 & 0 \\
0 & -\nu_{1} & 0 \\
0 & 0 & 1
\end{array}\right)+\varepsilon \boldsymbol{e}_{x}(\boldsymbol{q})\left(x, \frac{y_{\varepsilon}\left(x^{\prime}\right)}{\varepsilon}\right)
$$

hence, by (7) and (99)

$$
I_{3 \varepsilon}=|S| E_{T} \int \frac{\partial u_{\varepsilon 3}}{\partial x_{3}} \frac{\partial \mathfrak{v}_{\varepsilon 3}(\boldsymbol{\phi})}{\partial x_{3}} d m_{\varepsilon}+|\mathcal{S}| \varepsilon \int \frac{E_{T}}{1+\nu_{1}}\left(\frac{\nu_{1}}{1-2 \nu_{1}} \operatorname{tr}\left(\boldsymbol{e}\left(\boldsymbol{u}_{\varepsilon}\right)\right) \boldsymbol{I}+\boldsymbol{e}\left(\boldsymbol{u}_{\varepsilon}\right)\right): \boldsymbol{e}_{x}(\boldsymbol{q})\left(x, \frac{y_{\varepsilon}\left(x^{\prime}\right)}{\varepsilon}\right) d m_{\varepsilon}
$$

By Proposition 4, we have

$$
\begin{array}{r}
\left|\varepsilon \int \frac{E_{T}}{1+\nu_{1}}\left(\frac{\nu_{1}}{1-2 \nu_{1}} \operatorname{tr}\left(\boldsymbol{e}\left(\boldsymbol{u}_{\varepsilon}\right)\right) \boldsymbol{I}+\boldsymbol{e}\left(\boldsymbol{u}_{\varepsilon}\right)\right): \boldsymbol{e}_{x}(\boldsymbol{q})\left(x, \frac{y_{\varepsilon}\left(x^{\prime}\right)}{\varepsilon}\right) d m_{\varepsilon}\right| \\
\leq C \varepsilon \int\left|\frac{E_{T}}{1+\nu_{1}}\left(\frac{\nu_{1}}{1-2 \nu_{1}} \operatorname{tr}\left(\boldsymbol{e}\left(\boldsymbol{u}_{\varepsilon}\right)\right) \boldsymbol{I}+\boldsymbol{e}\left(\boldsymbol{u}_{\varepsilon}\right)\right)\right| d m_{\varepsilon} \\
\leq C \varepsilon \int\left|\boldsymbol{e}\left(\boldsymbol{u}_{\varepsilon}\right)\right| d m_{\varepsilon} \leq C \varepsilon \sqrt{\int\left|\boldsymbol{e}\left(\boldsymbol{u}_{\varepsilon}\right)\right|^{2} d m_{\varepsilon}} \leq C \varepsilon .
\end{array}
$$

By passing to the limit as $\varepsilon \rightarrow 0$ in (105), taking into account Proposition 4, (106) and the uniform convergence of $\left(\frac{\partial \mathfrak{v}_{\varepsilon 3}(\boldsymbol{\phi})}{\partial x_{3}}\right)$ to $\frac{\partial \phi_{3}}{\partial x_{3}}$ on $T_{\varepsilon}$, we obtain

$$
\lim _{\varepsilon \rightarrow 0} I_{3 \varepsilon}=|S| E_{T} \int_{\Sigma} \frac{\partial \gamma_{\Sigma}\left(u_{3}\right)}{\partial x_{3}} \frac{\partial \phi_{3}}{\partial x_{3}} d \mathcal{H}^{2} .
$$

We deduce from (99), (100), (101), and (107) that

$$
\lim _{\varepsilon \rightarrow 0} a_{\varepsilon, m}\left(\boldsymbol{u}_{\varepsilon}, \phi_{\varepsilon}\right)=a_{1}(\boldsymbol{u}, \boldsymbol{\phi}),
$$

where $a_{1}(.,$.$) is the bilinear form on \mathcal{D}_{1} \times \mathcal{D}_{1}$ defined by (22). Joining (98) and (108), we obtain

$$
a_{1}(\boldsymbol{u}, \boldsymbol{\phi})=\int_{\Omega} \boldsymbol{f} . \boldsymbol{\phi} d x \quad \forall \boldsymbol{\phi} \in \mathcal{D}\left(\Omega ; \mathbb{R}^{3}\right) .
$$

This variational formulation is equivalent to (10).

Case $m=3$. We choose $\phi_{3}=0$ and set (see Remark 2)

$$
\boldsymbol{\chi}_{\varepsilon}(x)=\left(\begin{array}{c}
\mathfrak{v}_{\varepsilon 1}(\boldsymbol{\phi})(x) \\
\mathfrak{v}_{\varepsilon 2}(\boldsymbol{\phi})(x) \\
0
\end{array}\right)+\varepsilon\left(\begin{array}{c}
0 \\
0 \\
-\frac{\partial \mathfrak{v}_{\varepsilon 1}(\boldsymbol{\phi})}{\partial x_{3}} \frac{y_{\varepsilon}\left(x^{\prime}\right)_{1}}{\varepsilon}-\frac{\partial \mathfrak{v}_{\varepsilon 2}(\boldsymbol{\phi})}{\partial x_{3}} \frac{y_{\varepsilon}\left(x^{\prime}\right)_{2}}{\varepsilon}
\end{array}\right)+\varepsilon^{2} \boldsymbol{q}\left(x, \frac{y_{\varepsilon}\left(x^{\prime}\right)}{\varepsilon}\right),
$$

where

$$
\boldsymbol{q}(x, y)=\nu_{1}\left(\begin{array}{c}
\frac{\partial^{2} \mathfrak{v}_{\varepsilon 1}(\boldsymbol{\phi})}{\partial x_{3}^{2}} \frac{y_{1}^{2}-y_{2}^{2}}{2}+\frac{\partial^{2} \mathfrak{v}_{\varepsilon 2}(\boldsymbol{\phi})}{\partial x_{3}^{2}} y_{1} y_{2} \\
\frac{\partial^{2} \mathfrak{v}_{\varepsilon 2}(\boldsymbol{\phi})}{\partial x_{3}^{2}} \frac{y_{2}^{2}-y_{1}^{2}}{2}+\frac{\partial^{2} \mathfrak{v}_{\varepsilon 1}(\boldsymbol{\phi})}{\partial x_{3}^{2}} y_{1} y_{2} \\
0
\end{array}\right)
$$


We have in $T_{\varepsilon}$

$$
\boldsymbol{e}\left(\boldsymbol{\chi}_{\varepsilon}\right)=\varepsilon\left(\frac{\partial^{2} \mathfrak{v}_{\varepsilon 1}(\boldsymbol{\phi})}{\partial x_{3}^{2}} \frac{y_{\varepsilon}\left(x^{\prime}\right)_{1}}{\varepsilon}+\frac{\partial^{2} \mathfrak{v}_{\varepsilon 2}(\boldsymbol{\phi})}{\partial x_{3}^{2}} \frac{y_{\varepsilon}\left(x^{\prime}\right)_{2}}{\varepsilon}\right)\left(\begin{array}{ccc}
\nu_{1} & 0 & 0 \\
0 & \nu_{1} & 0 \\
0 & 0 & -1
\end{array}\right)+\varepsilon^{2} \boldsymbol{e}_{x}(\boldsymbol{q})\left(x, \frac{y_{\varepsilon}\left(x^{\prime}\right)}{\varepsilon}\right)
$$

hence,

$$
\begin{aligned}
\frac{E_{T}}{1+\nu_{1}}\left(\frac{\nu_{1}}{1-2 \nu_{1}} \operatorname{tr}\left(\boldsymbol{e}\left(\boldsymbol{\chi}_{\varepsilon}\right)\right) \boldsymbol{I}+\boldsymbol{e}\left(\boldsymbol{\chi}_{\varepsilon}\right)\right)= & -\varepsilon\left(\frac{\partial^{2} \mathfrak{v}_{\varepsilon 1}(\boldsymbol{\phi})}{\partial x_{3}^{2}} \frac{y_{\varepsilon}\left(x^{\prime}\right)_{1}}{\varepsilon}+\frac{\partial^{2} \mathfrak{v}_{\varepsilon 2}(\boldsymbol{\phi})}{\partial x_{3}^{2}} \frac{y_{\varepsilon}\left(x^{\prime}\right)_{2}}{\varepsilon}\right) E_{T}\left(\boldsymbol{e}_{3} \otimes \boldsymbol{e}_{3}\right) \\
& +\varepsilon^{2} \frac{E_{T}}{1+\nu_{1}}\left(\frac{\nu_{1}}{1-2 \nu_{1}} \operatorname{tr}\left(\boldsymbol{e}_{x}(\boldsymbol{q})\left(x, \frac{y_{\varepsilon}\left(x^{\prime}\right)}{\varepsilon}\right)\right) \boldsymbol{I}+\boldsymbol{e}_{x}(\boldsymbol{q})\left(x, \frac{y_{\varepsilon}\left(x^{\prime}\right)}{\varepsilon}\right)\right) .
\end{aligned}
$$

Taking (20) into account, we infer

$$
\begin{aligned}
\frac{1}{\varepsilon^{m}} a_{T}\left(\boldsymbol{u}_{\varepsilon}, \boldsymbol{\chi}_{\varepsilon}\right) & =\frac{1}{\varepsilon^{3}} a_{T}\left(\boldsymbol{\chi}_{\varepsilon}, \boldsymbol{u}_{\varepsilon}\right)=\frac{1}{\varepsilon^{3}} \int_{T_{\varepsilon}}\left\{\frac{E_{T}}{1+\nu_{1}}\left(\frac{\nu_{1}}{1-2 \nu_{1}} \operatorname{tr}\left(\boldsymbol{e}\left(\boldsymbol{\chi}_{\varepsilon}\right)\right) \boldsymbol{I}+\boldsymbol{e}\left(\boldsymbol{\chi}_{\varepsilon}\right)\right): \boldsymbol{e}\left(\boldsymbol{u}_{\varepsilon}\right)\right\} d x \\
& =\frac{1}{\varepsilon^{3}} \int \varepsilon|S|\left\{\frac{E_{T}}{1+\nu_{1}}\left(\frac{\nu_{1}}{1-2 \nu_{1}} \operatorname{tr}\left(\boldsymbol{e}\left(\boldsymbol{\chi}_{\varepsilon}\right)\right) \boldsymbol{I}+\boldsymbol{e}\left(\boldsymbol{\chi}_{\varepsilon}\right)\right): \boldsymbol{e}\left(\boldsymbol{u}_{\varepsilon}\right)\right\} d m_{\varepsilon} \\
& =\int-|S| E_{T}\left(\frac{\partial^{2} \mathfrak{v}_{\varepsilon 1}(\boldsymbol{\phi})}{\partial x_{3}^{2}} \frac{y_{\varepsilon}\left(x^{\prime}\right)_{1}}{\varepsilon}+\frac{\partial^{2} \mathfrak{v}_{\varepsilon 2}(\boldsymbol{\phi})}{\partial x_{3}^{2}} \frac{y_{\varepsilon}\left(x^{\prime}\right)_{2}}{\varepsilon}\right)\left(\frac{1}{\varepsilon} \frac{\partial u_{\varepsilon 3}}{\partial x_{3}}\right) d m_{\varepsilon} \\
& +\int|S| \boldsymbol{e}\left(\boldsymbol{u}_{\varepsilon}\right): \frac{E_{T}}{1+\nu_{1}}\left(\frac{\nu_{1}}{1-2 \nu_{1}} \operatorname{tr}\left(\boldsymbol{e}_{x}(\boldsymbol{q})\left(x, \frac{y_{\varepsilon}\left(x^{\prime}\right)}{\varepsilon}\right)\right) \boldsymbol{I}+\boldsymbol{e}_{x}(\boldsymbol{q})\left(x, \frac{y_{\varepsilon}\left(x^{\prime}\right)}{\varepsilon}\right)\right) d m_{\varepsilon}
\end{aligned}
$$

Let us remark that $\boldsymbol{e}_{x}(\boldsymbol{q})\left(x, \frac{y_{\varepsilon}\left(x^{\prime}\right)}{\varepsilon}\right)$ is uniformly bounded on $T_{\varepsilon}$ and so we have, by the estimate (65) established in Proposition 4,

$$
\begin{gathered}
\left|\int \boldsymbol{e}\left(\boldsymbol{u}_{\varepsilon}\right): \frac{E_{T}}{1+\nu_{1}}\left(\frac{\nu_{1}}{1-2 \nu_{1}} \operatorname{tr}\left(\boldsymbol{e}_{x}(\boldsymbol{q})\left(x, \frac{y_{\varepsilon}\left(x^{\prime}\right)}{\varepsilon}\right)\right) \boldsymbol{I}+\boldsymbol{e}_{x}(\boldsymbol{q})\left(x, \frac{y_{\varepsilon}\left(x^{\prime}\right)}{\varepsilon}\right)\right) d m_{\varepsilon}\right| \\
\leq C \int\left|\boldsymbol{e}\left(\boldsymbol{u}_{\varepsilon}\right)\right| d m_{\varepsilon} \leq C \sqrt{\int\left|\boldsymbol{e}\left(\boldsymbol{u}_{\varepsilon}\right)\right|^{2} d m_{\varepsilon}} \leq C \sqrt{\varepsilon^{m-1}} \leq C \varepsilon \rightarrow 0
\end{gathered}
$$

By (25) and by the uniform continuity of $\boldsymbol{\phi}$ on $\bar{\Omega}$, the next estimate takes place:

$$
\left|\frac{\partial^{2} \mathfrak{v}_{\varepsilon \alpha}(\phi)}{\partial x_{3}^{2}}-\frac{\partial^{2} \phi_{\alpha}}{\partial x_{3}^{2}}\right|_{L^{\infty}\left(T_{\varepsilon}\right)} \leq C \varepsilon \quad \forall \alpha \in\{1,2\}
$$

Since, on the other hand, by Proposition 4 there holds $\int\left|\frac{1}{\varepsilon} \frac{\partial u_{\varepsilon 3}}{\partial x_{3}}\right|^{2} d m_{\varepsilon} \leq C$, taking (24) into account, we deduce that

$$
\begin{aligned}
& \mid \int\left(\left(\frac{\partial^{2} \mathfrak{v}_{\varepsilon 1}(\boldsymbol{\phi})}{\partial x_{3}^{2}} \frac{y_{\varepsilon 1}\left(x^{\prime}\right)}{\varepsilon}\right.\right.\left.\left.+\frac{\partial^{2} \mathfrak{v}_{\varepsilon 2}(\boldsymbol{\phi})}{\partial x_{3}^{2}} \frac{y_{\varepsilon 2}\left(x^{\prime}\right)}{\varepsilon}\right)-\left(\frac{\partial^{2} \phi_{1}}{\partial x_{3}^{2}} \frac{y_{\varepsilon 1}\left(x^{\prime}\right)}{\varepsilon}+\frac{\partial^{2} \phi_{2}}{\partial x_{3}^{2}} \frac{y_{\varepsilon 2}\left(x^{\prime}\right)}{\varepsilon}\right)\right)\left(\frac{1}{\varepsilon} \frac{\partial u_{\varepsilon 3}}{\partial x_{3}}\right) d m_{\varepsilon} \mid \\
& \leq C \varepsilon \int\left|\frac{1}{\varepsilon} \frac{\partial u_{\varepsilon 3}}{\partial x_{3}}\right| d m_{\varepsilon} \leq C \varepsilon\left(\int\left|\frac{1}{\varepsilon} \frac{\partial u_{\varepsilon 3}}{\partial x_{3}}\right|^{2} d m_{\varepsilon}\right)^{\frac{1}{2}}\left(m_{\varepsilon}(\Omega)\right)^{\frac{1}{2}} \leq C \varepsilon
\end{aligned}
$$


By Proposition 4 (see the first line of (70) and the last line of $(71)$ ), the sequence $\left(\frac{1}{\varepsilon} \frac{\partial u_{\varepsilon}}{\partial x_{3}}\right)$ two-scale converges with respect to $\left(m_{\varepsilon}\right)$ to $\frac{\partial w}{\partial x_{3}}(x)-\frac{\partial^{2} \gamma_{\Sigma}\left(u_{1}\right)}{\partial x_{3}^{2}}(x) y_{1}-\frac{\partial^{2} \gamma_{\Sigma}\left(u_{2}\right)}{\partial x_{3}^{2}}(x) y_{2}$. Therefore, by (1), (15), and (27), we have

$$
\begin{aligned}
& \lim _{\varepsilon \rightarrow 0} \int\left(\frac{\partial^{2} \phi_{1}}{\partial x_{3}^{2}} \frac{y_{\varepsilon 1}\left(x^{\prime}\right)}{\varepsilon}+\frac{\partial^{2} \phi_{2}}{\partial x_{3}^{2}} \frac{y_{\varepsilon 2}\left(x^{\prime}\right)}{\varepsilon}\right)\left(\frac{1}{\varepsilon} \frac{\partial u_{\varepsilon 3}}{\partial x_{3}}\right) d m_{\varepsilon} \\
& =\frac{1}{|S|} \iint_{\Sigma \times S}-|S| \frac{E_{T}}{2}\left(\frac{\partial^{2} \phi_{1}}{\partial x_{3}^{2}} y_{1}+\frac{\partial^{2} \phi_{2}}{\partial x_{3}^{2}} y_{2}\right)\left(\frac{\partial w}{\partial x_{3}}(x)-\frac{\partial^{2} \gamma_{\Sigma}\left(u_{1}\right)}{\partial x_{3}^{2}}(x) y_{1}-\frac{\partial^{2} \gamma_{\Sigma}\left(u_{2}\right)}{\partial x_{3}^{2}}(x) y_{2}\right) d \mathcal{H}^{2}(x) d y \\
& =|S| E_{T} \sum_{\alpha, \beta=1}^{2} \int_{\Sigma} J_{\alpha \beta} \frac{\partial^{2} \phi_{\alpha}}{\partial x_{3}^{2}} \frac{\partial^{2} \gamma_{\Sigma}\left(u_{\beta}\right)}{\partial x_{3}^{2}} d \mathcal{H}^{2}(x) .
\end{aligned}
$$

We deduce from (112), (113), (114), and (115) that

$$
\lim _{\varepsilon \rightarrow 0} I_{3 \varepsilon}=|S| E_{T} \sum_{\alpha, \beta=1}^{2} \int_{\Sigma} J_{\alpha \beta} \frac{\partial^{2} \phi_{\alpha}}{\partial x_{3}^{2}} \frac{\partial^{2} \gamma_{\Sigma}\left(u_{\beta}\right)}{\partial x_{3}^{2}} d \mathcal{H}^{2}(x) .
$$

By passing to the limit as $\varepsilon \rightarrow 0$ in (97), taking (98), (100), (101), and (116) into account, we obtain the variational formulation

$$
a_{0}(\boldsymbol{u}, \boldsymbol{\phi})+|S| \mid E_{T} \sum_{\alpha, \beta=1}^{2} \int_{\Sigma} J_{\alpha \beta} \frac{\partial^{2} \phi_{\alpha}}{\partial x_{3}^{2}} \frac{\partial^{2} \gamma_{\Sigma}\left(u_{\beta}\right)}{\partial x_{3}^{2}} d \mathcal{H}^{2}(x)=\int_{\Omega} \boldsymbol{f} . \boldsymbol{\phi} d x
$$

equivalent to (14).

\section{Other cases.}

If $0<m<1$, we simply set $\boldsymbol{\chi}_{\varepsilon}=\boldsymbol{\phi}$ (thus $\boldsymbol{\phi}_{\varepsilon}=\boldsymbol{\phi}$ ). Noticing that by and (65), we have

$$
\int_{T_{\varepsilon}}\left|\boldsymbol{e}\left(\boldsymbol{u}_{\varepsilon}\right)\right|^{2} d x \leq C \varepsilon^{m}
$$

we infer

$$
\begin{aligned}
\limsup _{\varepsilon \rightarrow 0}\left|I_{3 \varepsilon}\right| & =\limsup _{\varepsilon \rightarrow 0} \frac{1}{\varepsilon^{m}}\left|a_{T}\left(\boldsymbol{u}_{\varepsilon}, \boldsymbol{\phi}\right)\right| \\
& \left.\leq\left.\limsup _{\varepsilon \rightarrow 0} \frac{C}{\varepsilon^{m}}\left(\int_{T_{\varepsilon}} \mid \boldsymbol{e}\left(\boldsymbol{u}_{\varepsilon}\right)\right)\right|^{2} d x\right)^{\frac{1}{2}}\left|T_{\varepsilon}\right|^{\frac{1}{2}} \\
& \leq \limsup _{\varepsilon \rightarrow 0} C \varepsilon^{\frac{1-m}{2}}=0 .
\end{aligned}
$$

Joining (100), (101), (119), we obtain the variational formulation

equivalent to (8).

$$
a_{0}(\boldsymbol{u}, \boldsymbol{\phi})=\int_{\Omega} f \cdot \boldsymbol{\phi} d x
$$

If $1<m<3$, then by Proposition 4 we have $u_{3}=0$ on $\Sigma$. We define $\boldsymbol{\chi}_{\varepsilon}$ by (102), setting $\phi_{3}=0$. Since $\mathfrak{v}_{\varepsilon 3}(\boldsymbol{\phi})=0$, we deduce from (99) and (104) that

$$
\lim _{\varepsilon \rightarrow 0} I_{3 \varepsilon}=0 .
$$

The variational formulation obtained by passing to the limit in (97) as $\varepsilon \rightarrow 0$ is given by substituting 0 for $u_{3}$ and 0 for $\phi_{3}$ in (109).

If $m>3$, then by Proposition 4 we have $\boldsymbol{u}=0$ on $\Sigma$, and we set simply

$$
\chi_{\varepsilon}=0 \text {. }
$$


Remark 2 The field $\boldsymbol{\chi}_{\varepsilon}$ is constructed in such a way that the behavior of the couple $\left(\boldsymbol{\chi}_{\varepsilon}, \boldsymbol{e}\left(\boldsymbol{\chi}_{\varepsilon}\right)\right)$ should mimic that of $\left(\boldsymbol{u}_{\varepsilon}, \boldsymbol{e}\left(\boldsymbol{u}_{\varepsilon}\right)\right)$ in the fibres, studied in Proposition 4.

In the case $m=1$, the convergences (66) indicate that $\boldsymbol{e}\left(\boldsymbol{u}_{\varepsilon}\right) \simeq \boldsymbol{\Xi}_{0}\left(x, \frac{y_{\varepsilon}(x)}{\varepsilon}\right)$ in $T_{\varepsilon}$. This, joined with the relations (67) and (68), and by virtue of a minimization principle, suggests that the following approximation is likely to hold in $T_{\varepsilon}$

$$
\boldsymbol{e}\left(\boldsymbol{u}_{\varepsilon}\right) \simeq \frac{\partial u_{3}}{\partial x_{3}}(x) \boldsymbol{e}_{3} \otimes \boldsymbol{e}_{3}+\boldsymbol{M}\left(\frac{\partial u_{3}}{\partial x_{3}}(x)\right) \quad \text { in } T_{\varepsilon},
$$

where $\boldsymbol{M}(a)$ is the solution of

$$
\min _{\boldsymbol{M} \in \mathbb{S}^{3}, M_{33}=0} g\left(\boldsymbol{M}+a \boldsymbol{e}_{3} \otimes \boldsymbol{e}_{3}\right), \quad g(\boldsymbol{A}):=\frac{E_{T}}{2\left(1+\nu_{1}\right)}\left(\frac{\nu_{1}}{1-2 \nu_{1}}(\operatorname{tr} \boldsymbol{A})^{2}+\boldsymbol{A}: \boldsymbol{A}\right),
$$

given by

$$
M(a)=\left(\begin{array}{c}
-\nu_{1} a \\
-\nu_{1} a \\
0
\end{array}\right)
$$

Accordingly, the field $\boldsymbol{\chi}_{\varepsilon}$ we are looking for should satisfy

$$
\boldsymbol{\chi}_{\varepsilon} \simeq \boldsymbol{\phi} \quad \text { and } \quad \boldsymbol{e}\left(\boldsymbol{\chi}_{\varepsilon}\right) \simeq \frac{\partial \phi_{3}}{\partial x_{3}}(x) \boldsymbol{e}_{3} \otimes \boldsymbol{e}_{3}+\boldsymbol{M}\left(\frac{\partial \phi_{3}}{\partial x_{3}}(x)\right) \quad \text { in } T_{\varepsilon}
$$

These estimates are verified by $\chi_{\varepsilon}$ defined by (102), (103).

In the case $m=3$, the convergences (70) indicate that $\frac{1}{\varepsilon} \boldsymbol{e}\left(\boldsymbol{u}_{\varepsilon}\right) \simeq \boldsymbol{\Upsilon}_{0}\left(x, \frac{y_{\varepsilon}(x)}{\varepsilon}\right)$ in $T_{\varepsilon}$. The identification relations (71) can be improved, and further investigations show that in the linear isotropic case considered in this paper, there holds

$$
\boldsymbol{\Upsilon}_{0}(x, y) \simeq\left(-\frac{\partial^{2} \gamma_{\Sigma}\left(u_{1}\right)}{\partial x_{3}^{2}} y_{1}-\frac{\partial^{2} \gamma_{\Sigma}\left(u_{2}\right)}{\partial x_{3}^{2}} y_{2}\right) \boldsymbol{e}_{3} \otimes \boldsymbol{e}_{3}+\boldsymbol{e}_{y}\left(\boldsymbol{q}\left(\frac{\partial^{2} \gamma_{\Sigma}\left(u_{1}\right)}{\partial x_{3}^{2}}, \frac{\partial^{2} \gamma_{\Sigma}\left(u_{2}\right)}{\partial x_{3}^{2}}, y\right)\right)
$$

where $\boldsymbol{q}\left(a_{1}, a_{2},.\right)$ is the solution of

$$
\min _{\boldsymbol{q} \in H^{1}\left(S ; \mathbb{R}^{3}\right)} f_{S} g\left(\boldsymbol{e}_{y} \boldsymbol{q}+\left(-a_{1} y_{1}-a_{2} y_{2}\right) \boldsymbol{e}_{3} \otimes \boldsymbol{e}_{3}\right) d y, \quad g(\boldsymbol{A}):=\frac{E_{T}}{2\left(1+\nu_{1}\right)}\left(\frac{\nu_{1}}{1-2 \nu_{1}}(\operatorname{tr} \boldsymbol{A})^{2}+\boldsymbol{A}: \boldsymbol{A}\right)
$$

given by

$$
\boldsymbol{q}\left(a_{1}, a_{2}, y\right)=\nu_{1}\left(\begin{array}{c}
a_{1} \frac{y_{1}^{2}-y_{2}^{2}}{2}+a_{2} y_{1} y_{2} \\
a_{2} \frac{y_{2}^{2}-y_{1}^{2}}{2}+a_{1} y_{1} y_{2} \\
0
\end{array}\right)
$$

Accordingly, the field $\boldsymbol{\chi}_{\varepsilon}$ we are looking for should satisfy

$$
\chi_{\varepsilon 3} \simeq 0 \text { and } \frac{1}{\varepsilon} \boldsymbol{e}\left(\boldsymbol{\chi}_{\varepsilon}\right) \simeq\left(-\frac{\partial^{2} \gamma_{\Sigma}\left(u_{1}\right)}{\partial x_{3}^{2}} \frac{y_{\varepsilon 1}}{\varepsilon}-\frac{\partial^{2} \gamma_{\Sigma}\left(u_{2}\right)}{\partial x_{3}^{2}} \frac{y_{\varepsilon 2}}{\varepsilon}\right) \boldsymbol{e}_{3} \otimes \boldsymbol{e}_{3}+\boldsymbol{e}_{y}\left(\boldsymbol{q}\left(\frac{\partial^{2} \gamma_{\Sigma}\left(u_{1}\right)}{\partial x_{3}^{2}}, \frac{\partial^{2} \gamma_{\Sigma}\left(u_{2}\right)}{\partial x_{3}^{2}}, \frac{y_{\varepsilon}}{\varepsilon}\right)\right)
$$

These estimates are verified by $\chi_{\varepsilon}$ defined by (110). 


\subsection{Strong convergence}

To fix the ideas, we assume $m=1$ (the other cases are similar). The space $\mathcal{D}_{1}$ defined by (11), equipped with the inner product $a_{1}(.,$.$) defined by (22)$, is a Hilbert space in which $\mathcal{D}\left(\Omega ; \mathbb{R}^{3}\right)$ is dense. Hence, fixing $\eta>0$, we can choose $\phi \in \mathcal{D}\left(\Omega ; \mathbb{R}^{3}\right)$ such that

$$
a_{1}(\boldsymbol{u}-\boldsymbol{\phi}, \boldsymbol{u}-\boldsymbol{\phi})<\eta
$$

and consider $\phi_{\varepsilon}$ defined by (96). There holds (see (19))

$$
\begin{aligned}
\left|\boldsymbol{u}_{\varepsilon}-\boldsymbol{\phi}_{\varepsilon}\right|_{H_{0}^{1}\left(\Omega ; \mathbb{R}^{3}\right)}^{2} \leq C a_{\varepsilon, m}\left(\boldsymbol{u}_{\varepsilon}-\boldsymbol{\phi}_{\varepsilon}, \boldsymbol{u}_{\varepsilon}-\boldsymbol{\phi}_{\varepsilon}\right) & \\
& =C(\underbrace{a_{\varepsilon, m}\left(\boldsymbol{u}_{\varepsilon}, \boldsymbol{u}_{\varepsilon}\right)}_{J_{1 \varepsilon}}-2 \underbrace{a_{\varepsilon, m}\left(\boldsymbol{u}_{\varepsilon}, \boldsymbol{\phi}_{\varepsilon}\right)}_{J_{2 \varepsilon}}+\underbrace{a_{\varepsilon, m}\left(\boldsymbol{\phi}_{\varepsilon}, \boldsymbol{\phi}_{\varepsilon}\right)}_{J_{3 \varepsilon}}) .
\end{aligned}
$$

Since $\boldsymbol{u}_{\varepsilon}$ is the solution of (7), we have $J_{1 \varepsilon}=\int_{\Omega} \boldsymbol{f} . \boldsymbol{u}_{\varepsilon} d x$. We then deduce from the weak convergence of $\boldsymbol{u}_{\varepsilon}$ to $\boldsymbol{u}$ in $H_{0}^{1}\left(\Omega ; \mathbb{R}^{3}\right)$ established in Proposition 4 , that

$$
\lim _{\varepsilon \rightarrow 0} J_{1 \varepsilon}=\int_{\Omega} \boldsymbol{f} \cdot \boldsymbol{u} d x=a_{1}(\boldsymbol{u}, \boldsymbol{u})
$$

By (108), there holds

$$
\lim _{\varepsilon \rightarrow 0} J_{2 \varepsilon}=a_{1}(\boldsymbol{u}, \boldsymbol{\phi})
$$

A straightforward computation yields

$$
\lim _{\varepsilon \rightarrow 0} J_{3 \varepsilon}=a_{1}(\boldsymbol{\phi}, \boldsymbol{\phi}) .
$$

Collecting (123), (124), (125), (126), and (127), we obtain

$$
\limsup _{\varepsilon \rightarrow 0}\left|\boldsymbol{u}_{\varepsilon}-\boldsymbol{\phi}_{\varepsilon}\right|_{H_{0}^{1}\left(\Omega ; \mathbb{R}^{3}\right)}^{2} \leq C a_{1}(\boldsymbol{u}-\boldsymbol{\phi}, \boldsymbol{u}-\boldsymbol{\phi}) \leq C \eta .
$$

It is easy to check that

$$
\begin{aligned}
& \limsup _{\varepsilon \rightarrow 0}\left|\boldsymbol{\phi}-\boldsymbol{\phi}_{\varepsilon}\right|_{H_{0}^{1}\left(\Omega ; \mathbb{R}^{3}\right)}=0, \\
& |\boldsymbol{u}-\boldsymbol{\phi}|_{H_{0}^{1}\left(\Omega ; \mathbb{R}^{3}\right)}^{2} \leq C a_{1}(\boldsymbol{u}-\boldsymbol{\phi}, \boldsymbol{u}-\boldsymbol{\phi}) \leq C \eta
\end{aligned}
$$

We infer from (131) and (129) that

$$
\begin{aligned}
\limsup _{\varepsilon \rightarrow 0}\left|\boldsymbol{u}-\boldsymbol{u}_{\varepsilon}\right|_{H_{0}^{1}\left(\Omega ; \mathbb{R}^{3}\right)} & \leq \limsup _{\varepsilon \rightarrow 0}|\boldsymbol{u}-\boldsymbol{\phi}|_{H_{0}^{1}\left(\Omega ; \mathbb{R}^{3}\right)}+\left|\boldsymbol{\phi}-\boldsymbol{\phi}_{\varepsilon}\right|_{H_{0}^{1}\left(\Omega ; \mathbb{R}^{3}\right)}+\left|\boldsymbol{\phi}_{\varepsilon}-\boldsymbol{u}_{\varepsilon}\right|_{H_{0}^{1}\left(\Omega ; \mathbb{R}^{3}\right)} \\
& \leq C \sqrt{\eta}
\end{aligned}
$$

By the arbitrary choice of $\eta$, the strong convergence of $\left(\boldsymbol{u}_{\varepsilon}\right)$ to $\boldsymbol{u}$ in $H_{0}^{1}\left(\Omega ; \mathbb{R}^{3}\right)$ is proved.

Remark 3 In view of (124-127), we established indeed that

$$
\limsup _{\varepsilon \rightarrow 0} a_{\varepsilon, m}\left(\boldsymbol{u}_{\varepsilon}-\phi_{\varepsilon}, \boldsymbol{u}_{\varepsilon}-\phi_{\varepsilon}\right) \leq C a_{1}(\boldsymbol{u}-\boldsymbol{\phi}, \boldsymbol{u}-\boldsymbol{\phi}),
$$

for all field $\boldsymbol{\phi}$ that can be used in the construction of the sequence of oscillating test fields $\left(\phi_{\varepsilon}\right)$ (defined by (96)). Looking back at the proof of the weak convergence, we notice that the proof remains unchanged if we only assume, instead of $\phi \in \mathcal{D}\left(\Omega ; \mathbb{R}^{3}\right)$, that $\phi$ satisfies

$$
\phi \in C\left(\bar{\Omega} ; \mathbb{R}^{3}\right) \cap C^{1}\left(\bar{\Omega}^{+} ; \mathbb{R}^{3}\right) \cap C^{1}\left(\bar{\Omega}^{-} ; \mathbb{R}^{3}\right) ; \quad \frac{\partial \phi}{\partial x_{3}} \in C\left(\bar{\Omega} ; \mathbb{R}^{3}\right) ; \quad \boldsymbol{\phi}=0 \quad \text { on } \partial \Omega,
$$


and

$$
\frac{\partial^{2} \phi_{\alpha}}{\partial x_{3}^{2}} \in C\left(\bar{\Omega} ; \mathbb{R}^{3}\right) \text { for } \alpha \in\{1,2\} \quad \text { if } m=3 .
$$

Due to the discontinuity of $\boldsymbol{\sigma}_{0} \boldsymbol{e}_{1}$ across $\Sigma$ (see (10), (12)), we can not expect the derivative with respect to $x_{1}$ of the solution $\boldsymbol{u}$ of the limit problem to be continuous on $\bar{\Omega}$. However, for a sufficiently regular datum $f$, we can expect that

$$
\boldsymbol{u} \text { satisfies (132) and (133). }
$$

Under (134), we can substitute $\boldsymbol{u}$ for $\boldsymbol{\phi}$ in the definition of $\boldsymbol{\phi}_{\varepsilon}$. Let us denote by $\widetilde{\boldsymbol{u}}_{\varepsilon}$ the field obtained in this way (to avoid the confusion with the solution $\boldsymbol{u}_{\varepsilon}$ of the elasticity problem (7)). We then deduce from (131) that

$$
\limsup _{\varepsilon \rightarrow 0} a_{\varepsilon, m}\left(\boldsymbol{u}_{\varepsilon}-\widetilde{\boldsymbol{u}}_{\varepsilon}, \boldsymbol{u}_{\varepsilon}-\widetilde{\boldsymbol{u}}_{\varepsilon}\right)=0 .
$$

In particular, by the definition (19) of $a_{\varepsilon, m}$, we get, since $\widetilde{\boldsymbol{u}}_{\varepsilon}=\widetilde{\boldsymbol{\chi}}_{\varepsilon}$ on $T_{\varepsilon}$, denoting by $\widetilde{\boldsymbol{\chi}}_{\varepsilon}$ the field deduced by substituting $\boldsymbol{u}$ for $\boldsymbol{\phi}$ in (102), (110):

$$
\limsup _{\varepsilon \rightarrow 0} \frac{1}{\varepsilon^{m}} \int_{T_{\varepsilon}}\left\{\frac{E_{T}}{1+\nu_{1}}\left(\frac{\nu_{1}}{1-2 \nu_{1}} \operatorname{tr}\left(\boldsymbol{e}\left(\boldsymbol{u}_{\varepsilon}-\widetilde{\boldsymbol{\chi}}_{\varepsilon}\right)\right) \boldsymbol{I}+\boldsymbol{e}\left(\boldsymbol{u}_{\varepsilon}-\widetilde{\boldsymbol{\chi}}_{\varepsilon}\right)\right): \boldsymbol{e}\left(\boldsymbol{u}_{\varepsilon}-\widetilde{\boldsymbol{\chi}}_{\varepsilon}\right)\right\} d x=0 .
$$

This implies, since $\left|T_{\varepsilon}\right| \simeq \varepsilon$, that

$$
\limsup _{\varepsilon \rightarrow 0} \frac{1}{\varepsilon^{m-1}} f_{T_{\varepsilon}}\left|\boldsymbol{e}\left(\boldsymbol{u}_{\varepsilon}-\widetilde{\boldsymbol{\chi}}_{\varepsilon}\right)\right|^{2} d x=0 .
$$

If $m=1$, it follows from (104) that

$$
\limsup _{\varepsilon \rightarrow 0} f_{T_{\varepsilon}}\left|\boldsymbol{e}\left(\boldsymbol{u}_{\varepsilon}\right)-\frac{\partial u_{3}}{\partial x_{3}}\left(\begin{array}{ccc}
-\nu_{1} & 0 & 0 \\
0 & -\nu_{1} & 0 \\
0 & 0 & 1
\end{array}\right)\right|^{2} d x=0
$$

justifying (121).

If $m=3$, we deduce from (111) and (135) that

$$
\limsup _{\varepsilon \rightarrow 0} f_{T_{\varepsilon}}\left|\frac{1}{\varepsilon} \boldsymbol{e}\left(\boldsymbol{u}_{\varepsilon}\right)-\left(\frac{\partial^{2} u_{1}}{\partial x_{3}^{2}} \frac{y_{\varepsilon}\left(x^{\prime}\right)_{1}}{\varepsilon}+\frac{\partial^{2} u_{2}}{\partial x_{3}^{2}} \frac{y_{\varepsilon}\left(x^{\prime}\right)_{2}}{\varepsilon}\right)\left(\begin{array}{ccc}
\nu_{1} & 0 & 0 \\
0 & \nu_{1} & 0 \\
0 & 0 & -1
\end{array}\right)\right|^{2} d x=0,
$$

which shows that

$$
\frac{1}{\varepsilon} \boldsymbol{e}\left(\boldsymbol{u}_{\varepsilon}\right) \simeq\left(\frac{\partial^{2} u_{1}}{\partial x_{3}^{2}} \frac{y_{\varepsilon}\left(x^{\prime}\right)_{1}}{\varepsilon}+\frac{\partial^{2} u_{2}}{\partial x_{3}^{2}} \frac{y_{\varepsilon}\left(x^{\prime}\right)_{2}}{\varepsilon}\right)\left(\begin{array}{ccc}
\nu_{1} & 0 & 0 \\
0 & \nu_{1} & 0 \\
0 & 0 & -1
\end{array}\right) \quad \text { in } \quad T_{\varepsilon},
$$

in agrement with (122).

\section{Concluding remarks and open problems}

1. The comparison of the results of Theorem 1 with some of the results obtained in [11], [12] and [13] for the case of an homogeneous layer is interesting: indeed in theses cases $\Sigma$ behaves as a "material surface" of plate-like type with membrane Kirchhoff-Love energy for $m=1$ and with bending energy for $m=3$. In the present case Theorem 1 means that $\Sigma$ always behaves as a "material surface". For $m=1$ the "material surface" is without membrane energy in the direction of the plane $\Sigma$ orthogonal to the direction of the fibres. For $m=3$ the "material surface" $\Sigma$ is without bending energy in the direction orthogonal to the fibres. In the papers [12] and [13] it is also considered the more general situation of a surface $\Sigma$ and of a shell-like inclusion; the results obtained in this situation suggest to study the case of fibres that 
are tubular neighbourhoods of a family of lines of principal curvature of the surface $\Sigma$.

2. Here we have considered the case of isotropic materials and of fibres with "constant" section. In this case the study of asymptotic theory of slender beams gives models where torsion and traction are uncoupled. The case of anisotropic materials and/or fibres whose section "varies with $\varepsilon$ " deserves special attention. Indeed in this case asymptotic theory of slender beams can present coupling phenomena between torsion and traction ( see, e.g., [18], [24], [25]), or non-local effects (as in homogenization, see, e.g., [18], [25], [4], [9]). A research in this direction is actually done by the first author (M. B.).

3. The transmission conditions on $\Sigma$ imply that some "singular behaviour" can appear there and at $\partial \Sigma \cap \partial \Omega$; these "singular behaviours" could be analysed for instance with the methods of [19].

Acknowledgement. The work of G.G. and F.K. has been partially supported by the French Agence Nationale de la Recherche (ANR) under Grant ARAMIS (Projet "Blanc", ANR 12 BS01-0021) (Analysis of Robust Asymptotic Methods In numerical Simulation in mechanics).

\section{References}

1. R. Abdelmoula, J. J. Marigo: The effective behavior of a fiber bridged crack, J. Mech. Phys. Solids, 48 (2000), pp. 2419-2444.

2. G. Allaire: Homogenization and two-scale convergence, SIAM J. Math. Anal., 23 (1992), pp. $1482-$ 1518.

3. T. Arbogast, J. Douglas, U. Hornung: Derivation of the double porosity model of single phase flow via homogenization theory, SIAM J. Math. Anal., 21 (1990), pp. 823-836.

4. M. Bellieud : Torsion effects in elastic composites with high contrast, SIAM J. Math. Anal., 41 (2010), pp. 2514-2553.

5. M. Bellieud: A notion of capacity related to elasticity. Applications to homogenization, Arch. Ration. Mech. Anal., 203 (2012), pp.137-187.

6. M. Bellieud: Problèmes capacitaires en viscoplasticité avec effets de torsion, C. R. Acad. Sci. Paris, Ser. I, 351 (2013), pp. 241-245.

7. M. Bellieud, G. Bouchitté: Homogenization of a soft elastic material reinforced by fibres, Asymptotic Analysis, 32 ( 2002), pp. 153-183.

8. G. Bouchitté, I. Fragalà: Homogenization of thin structures by two-scale method with respect to measures, SIAM J. Math. Anal., 32 (2001), p. 1198-1226

9. M. Bellieud, I. Gruais: Homogenization of an elastic material reinforced by very stiff or heavy fibers. Non local effects. Memory effects, J. Math. Pures Appl., 84 (2005), pp. 55-96.

10. A. Bensoussan, J.-L. Lions, G. Papanicolaou: Asymptotic analysis for periodic structures, AMS Chelsea Publishing, Providence, RI, 2011, corrected reprint of the 1978 original

11. A. L. Bessoud, F. Krasucki, G. Michaille: Multi-materials with strong interface: variational modelings, Asymptotic Analysis, 61 (2009), pp. 1-19.

12. A.L. Bessoud, F. Krasucki, M. Serpilli: Plate-like and shell-like inclusions with high rigidity, $C . R$. Acad. Sci. Paris, Ser. I, 346 (2008), pp. 697-702.

13. A. L. Bessoud, F. Krasucki, M. Serpilli: Asymptotic analysis of shell-like inclusions with high rigidity, Journal of Elasticity, 103 (2011), pp. 153-172.

14. K. B. Cherednichenko, M. Cherdantsev: Two-scale $\Gamma$-convergence of integral functionals and its application to homogenisation of nonlinear high-contrast periodic composites, Arch. Ration. Mech. Anal., 204 (2012), pp. 445-478.

15. D.Cioranescu, A. Damlamian, G. Griso: The periodic unfolding method in homogenization, SIAM J. Math. Anal., 40 (2008), pp. 1585-1620.

16. D. Cioranescu, P. Donato: An introduction to homogenization, Oxford Lecture Series in Mathematics and its Applications, Vol. 17, Oxford University Press, New York, 1999.

17. G. Geymonat, F. Krasucki, S. Lenci: Bonded joint with a soft thin adhesive, Mathematics and Mechanics of Solids, 4 (1999) 201-225. 
18. G. Geymonat, F. Krasucki, J.J. Marigo : Stress distribution in anisotropic elastic composite beams, Applications of Multiple Scaling in Mechanics, P.G. Ciarlet and E. Sanchez-Palencia ed., Masson, 1987, pp. 118-133

19. P. Grisvard: Singularities in Boundary Values Problems, Masson-Springer Verlag, 1992,

20. F. Krasucki, S. Lenci: Analysis of interfaces of variable stiffness, Intern. J. of Solids and Structures, 37 (2000), pp. 3619-3632.

21. E. Y. Khruslov: Homogenized models of composite media, Birkhäuser 1991.

22. D. Lukkassen, G. Nguetseng, P. Wall: Two-scale convergence, Int. J. of Pure and Appl. Math., 2 (2002), pp. 35-86

23. D. Lukkassen, P. Wall: Two-scale convergence with respect to measures and homogenization of monotone operators, J. Funct. Spaces Appl., 3 (2005), pp. 125-161.

24. F. Murat, A. Sili: Comportement asymptotique des solutions du système de l'élasticité linéarisée anisotrope hétérogène dans des cylindres minces, C. R. Acad. Sci. Paris, Ser. I, 328 (1999), pp. 179-184.

25. F. Murat, A. Sili: Effets non locaux dans le passage 3d-1d en élasticité linéarisée anisotrope hétérogène, C. R. Acad. Sci. Paris, Ser. I, 330 (2000), pp. 745-750.

26. J.J. Marigo, C. Pideri: The effective behavior of elastic bodies containing microcracks or microholes localized on a surface, International Journal of Damage Mechanics, 20 (2011), pp. 1130-1150.

27. S. Neukamm: Rigorous derivation of a homogenized bending-torsion theory for inextensible rods from 3d elasticity. Arch. Ration. Mech. Anal., 206 (2012), pp. 645-706.

28. G. Nguetseng: A general convergence result for a functional related to the theory of homogenization, SIAM J. Math. Anal., 20 (1989), pp. 608-623.

29. G. Nguetseng, E. Sanchez-Palencia: Stress concentration for defects distributed near a surface, Local Effects in the Analysis of Structures, P. Ladevèze ed., Elsevier, Amsterdam, 1986, pp. 55-74.

30. O. A. Oleinik, A. S. Shamaev, G. A. Yosifian: Mathematical problems in elasticity and homogenization, North-Holland Publishing Co., Amsterdam, 1992.

31. C. Pideri, P. Seppecher: A second gradient material resulting from the homogenization of an heterogeneous linear elastic medium, Contin. Mech. Thermodyn., 9 (1997), pp. 241-257.

32. E. Sanchez Palencia: Non-homogeneous media and vibration theory, Springer Verlag, Berlin, 1980.

33. P. E. Stelzig: On problems in homogenization and two-scale convergence, $\mathrm{PhD}$ thesis, University of Trento, April 2012

34. L. Tartar: Cours Peccot, Collège de France, (1977), unpublished, partially written in F. Murat, L. Tartar: H-convergence, Topics in the Mathematical Modelling of Composite Materials, L. Cherkaevand R. V. Kohn ed, Progress in Nonlinear Differential Equations and their Applications, 31, Birkaüser, Boston (1998), pp. 21-43.

35. A. Visintin: Toward a two-scale calculus, ESAIM Control Optim. Calc. Var., 12 (2006), pp. 371-397.

36. V. V. Zhikov: On an extension and an application of the two-scale convergence method. (Russian) Mat. Sb., 191 (2000), no. 7, pp. 31-72; English translation in Sb. Math., 191 (2000), no. 7, pp. 973-1014. 